\title{
¿Requiere la interferencia de la superposición de ondas como principio físico?
}

\author{
Román Castañeda \\ Escuela de Física, Universidad Nacional de Colombia Sede Medellín, Medellín, Colombia \\ Conferencia de posesión para el ingreso como miembro de Número de la \\ Academia Colombiana de Ciencias Exactas, Físicas y Naturales el 23 de noviembre de 2017
}

\begin{abstract}
Resumen
La superposición de ondas es una de las ideas fundamentales más preciadas de la física. Durante cerca de dos siglos ha constituido el principio físico de la interferencia y la difracción de las ondas y, a lo largo de los últimos cien años se ha empleado como instrumento predictivo del comportamiento de sistemas cuánticos. Sin embargo, dicha superposición no puede explicar la interferencia de las ondas y de las partículas de manera generalizada debido, principalmente, a la diferente interpretación de la función de onda en cada contexto. Dado que los fenómenos del mismo tipo deben obedecer al mismo principio, o ser consecuencia de las mismas causas, esta limitación de la superposición de ondas es, por lo tanto, un problema actual en los fundamentos de la física. Como solución se propone un nuevo principio físico, el cual proporciona la misma explicación para la interferencia de las ondas y de las partículas sin detrimento de la precisión predictiva. Su validez se apoya en el análisis de resultados experimentales reportados por otros autores. Este principio es completamente novedoso y conduce a una formulación no estandarizada de la interferencia, que deberá abrir nuevos campos de discusión sobre este tema fundamental de la física. (C) 2017. Acad. Colomb. Cienc. Ex. Fis. Nat.
\end{abstract}

Palabras clave: Interferencia; Superposición de ondas; Correlación; Espectro de clases.

Does interference require the wave superposition as physical principle?

\begin{abstract}
The wave superposition is one of the most precious foundations of physics. It has been the physical principle of interference and diffraction of waves for nearly two centuries, and an accurate predictive instrument of the behavior of quantum systems over the past 100 years. However, it cannot explain the interference of waves and particles in a generalized way mainly due to the different interpretation of the wave function in each context. Given that phenomena of the same type should obey to the same principle or be the consequence of the same causes, this restriction of the wave superposition constitutes a current problem in the very foundations of physics. A new physical principle is introduced to address this problem. It provides the same explanation for wave and particle interference, but maintaining the predictive accuracy of the wave superposition. Its validity is based on the analysis of experimental results reported by other authors. This principle is completely new and leads to a non-standardized formulation of interference, which should open new avenues of discussion on this fundamental topic of physics. (C) 2017. Acad. Colomb. Cienc. Ex. Fis. Nat.
\end{abstract}

Key words: Interference; Wave superposition; Two-point correlation; Spectrum of classes.

\section{Introducción}

La superposición de ondas es una de las nociones más arraigadas de la física. A inicios del siglo 19 fue introducida como principio físico de la interferencia de la luz por Thomas Young (Born \& Wolf, 1993), y a partir de la interpretación probabilística de la función de onda cuántica, introducida por Max Born en la segunda década del siglo 20 (Feynman, et al., 1965), se utiliza como herramienta matemática para predecir la formación de patrones de interferencia de las partículas. Así, a lo largo de los dos últimos siglos, esta eficacia de la superposición de ondas ha logrado que su validez, relacionada con el fenómeno de interferencia, se acepte sin cuestionamientos.

Sin embargo, aunque la capacidad predictiva de una noción física es una condición necesaria para establecer su validez, no es una condición suficiente para erigirla como

\footnotetext{
Correspondencia:

Román Castañeda, rcastane@unal.edu.co

Recibido: 08 de agosto de 2017

Aceptado: 6 de diciembre de 2017
} 
principio. Para ello se requiere también una probada capacidad explicativa de los fenómenos. En el caso de la superposición de ondas, existe un claro contraste entre su bien establecida capacidad predictiva y sus restricciones explicativas. En efecto, la superposición de ondas puede explicar fenomenológicamente y predecir matemáticamente la interferencia de ondas clásicas (Born \& Wolf, 1993), pero solo puede predecir matemáticamente la interferencia de partículas masivas como electrones, átomos y moléculas. Por ello, Richard Feynman afirmó que la interferencia de Young con electrones individuales “... contiene el corazón de la mecánica cuántica. En realidad, encierra su único misterio” (Feynman \& Hibbs, 1965).

Se han utilizado diferentes estrategias experimentales con el fin de registrar patrones de interferencia con partículas, y se han intentado varias descripciones orientadas a explicar los resultados fenomenológicamente (Zeilinger, et al., 1988; Arndt, et al., 1999; Nairz, et al., 2003; Juffmann, et al., 2009; Frabboni, et al., 2012; Juffmann, et al., 2012; Matteucci, et al., 2013; Bach, et al., 2013; Castañeda, et al., 2016 a, b). Dichas descripciones se basan en la mecánica cuántica y algunas de ellas pueden incluir argumentos 'semiclásicos', pues se supone que la interferencia de partículas no puede explicarse enteramente a partir de la mecánica clásica. Por el contrario, en el caso de la interferencia de ondas clásicas, la superposición de ondas aporta una única y eficaz explicación fenomenológica, lo que le da el estatus de principio físico.

No se ha establecido, por lo tanto, un principio único y general que explique de igual manera la interferencia tanto de ondas clásicas como de partículas masivas. En este contexto, las reglas de la filosofía natural de Isaac Newton (Newton, 1686) delinean un reto epistemológico importante y actual, pues establecen que, (i) no se deben admitir otras causas que las necesarias para explicar los fenómenos, y (ii) los efectos del mismo género deben siempre ser atribuidos, en la medida en que sea posible, a la misma causa. En este artículo se establece cómo la teoría clásica de la correlación espacial (Mandel \& Wolf, 1995) ofrece un marco idóneo para la formulación de un principio que cumple con los requerimientos del reto. Dicho principio explica la interferencia como el resultado de interacciones individuales entre "emisores puntuales reales", que representan la perturbación de la onda o la partícula que atraviesa una abertura dada en la máscara de interferencia, y "emisores puntuales virtuales" determinados por la configuración del montaje experimental. Se muestra, así mismo, que la validez de este nuevo principio implica que la superposición de ondas no es una condición necesaria para describir y predecir la interferencia.

El ejercicio de sintetizar el principio requiere importantes modificaciones de la interpretación convencional del modelo matemático. En la sección 2 de esta presentación, la expansión modal de la correlación espacial se interpreta como una condición geométrica en el volumen delimitado por el montaje experimental. En la sección 3, se muestra que los emisores puntuales virtuales determinan un "potencial geométrico" en el espacio donde se mueven las ondas o las partículas, y se introduce una nueva ley general de interferencia. El "espectro de clases de emisores puntuales" se presenta en la sección 4 como un instrumento que permite construir el mapa completo de las interacciones de un experimento de interferencia dado. También se discute la predicción exacta de las modulaciones de difracción y la influencia de la incertidumbre en la posición de los emisores puntuales reales en interferencia de partículas singulares (en este tipo de experimentos se emiten partículas individuales de tal manera que la emisión de una de ellas ocurre después de que la anterior ha sido registrada por el detector). La validez de este desarrollo se respalda en el análisis de patrones de interferencia experimentales con moléculas individuales reportado por otros autores (Castañeda, et al., 2016 a, b). Ya que la teoría que se discute a continuación introduce nuevos términos y conceptos no estandarizados, se espera con ello abrir nuevos campos de discusión sobre este tema fundamental de la física.

\section{Interpretaciones novedosas}

Para describir formalmente un experimento de interferencia con ondas o con partículas es necesario considerar dos escenarios, a saber: (i) el modelo teórico y (ii) la configuración del arreglo experimental. El modelo teórico se basa en las partes temporalmente independientes de la ecuación de ondas de Maxwell, en el caso de ondas clásicas (Born \& Wolf, 1993), y de la ecuación de Schrödinger, en el de las partículas masivas (Feynman, et al., 1965). Para sintetizar el nuevo principio de interferencia es suficiente considerar que las ondas y las partículas se mueven en el espacio libre, con la única limitación de las condiciones de frontera impuestas por la configuración del montaje. Así, las partes temporalmente independientes de ambas ecuaciones tendrán la misma forma matemática dada por la ecuación de Helmholtz $\nabla^{2} \psi(\mathbf{r})+k^{2} \psi(\mathbf{r})=0$, donde $\psi(\mathbf{r})$ y $-k^{2}$ son las funciones y los valores propios del operador laplaciano. El vínculo del valor propio con parámetros físicos de las ondas o de las partículas, según el caso, determina la especificidad de la ecuación de Helmholtz. Así, (i) $k=\omega / c$, si la ecuación de Helmholtz se refiere a ondas clásicas de frecuencia $\omega$ y velocidad $c$; (ii) $k=E / \hbar c$, si se refiere a fotones de energía $E$, con $\hbar=h / 2 \pi$, siendo $h$ la constante de Planck, y (iii) $k=p / \hbar$, si se refiere a partículas masivas de momento $p$.

No obstante, las funciones propias del operador laplaciano no son observables físicos en general, pues los detectores que se emplean en los experimentos de interferencia son de módulo cuadrático, es decir, dispositivos que registran la distribución de energía de la onda o de las partículas que inciden sobre el plano del detector. Para determinar este observable físico es necesario considerar la correlación de las funciones propias en parejas de puntos en el espacio, expresada como $W\left(\mathbf{r}_{1}, \mathbf{r}_{2}\right)=\left\langle\psi\left(\mathbf{r}_{1}\right) \psi^{*}\left(\mathbf{r}_{2}\right)\right\rangle$, donde el símbolo \langle\rangle representa el promedio de ensamble y el asterisco, el complejo conjugado (Mandel \& Wolf, 
1995). Dicha correlación es solución de dos ecuaciones de Helmholtz acopladas por su valor propio, expresadas como $\nabla_{j}^{2} \psi\left(\mathbf{r}_{j}\right)+k^{2} \psi\left(\mathbf{r}_{j}\right)=0$, con $j=1,2$ (Mandel \& Wolf, 1995). En efecto, el observable físico registrado por el detector es la componente de la correlación para $\mathbf{r}_{1}=\mathbf{r}_{2}=\mathbf{r}$, esto es $S(\mathbf{r})=W(\mathbf{r}, \mathbf{r})=\left\langle|\psi(\mathbf{r})|^{2}\right\rangle$. Así, la descripción teórica de un experimento de interferencia con ondas o con partículas consiste en establecer las funciones de correlación asociadas a la configuración del arreglo experimental.

El segundo escenario, esto es la configuración del arreglo experimental, determina las condiciones de frontera requeridas. La Figura 1 indica que dicho arreglo está compuesto, en general, por dos etapas conectadas en cascada y denotadas respectivamente como SM (del plano de la fuente $\mathrm{S}$, al plano de la máscara $\mathrm{M}$, separados por una distancia $\mathrm{z}^{\prime}$ ) y $\mathrm{MD}$ (del plano $\mathrm{M}$ al plano del detector $\mathrm{D}$, separados por una distancia $z$ ). La etapa SM se denomina también "etapa de preparación" porque especifica las distribuciones de energía y correlación sobre el plano $\mathrm{M}$, para que la interferencia se produzca en la "etapa de realización” MD, y la distribución de energía del patrón de interferencia pueda ser registrada por el detector. Por lo tanto, la descripción teórica completa del experimento se logra estableciendo la función de correlación de pares de puntos en el plano $\mathrm{M}$ y la distribución de energía en el plano D. En la sección A de la Información suplementaria se indica el procedimiento matemático que permite expresar, (i) la correlación en el plano M como una expansión modal en la etapa de preparación, cuyos coeficientes son los valores de la correlación en el plano S, y (ii) la distribución de energía en el plano D como una expansión modal en la etapa de realización, cuyos coeficientes son los valores de la correlación en el plano $\mathrm{M}$.

Es útil expresar dichas expansiones modales en función de las coordenadas reducidas $\left(\mathbf{r}_{A}^{\prime}, \mathbf{r}_{D}^{\prime}\right),\left(\xi_{A}, \xi_{D}\right)$ y $\left(\mathbf{r}_{A}, \mathbf{r}_{D}\right)$ ilustradas en la Figura 1, las cuales determinan unívocamente parejas de puntos en los planos S, M y D, respectivamente, señalizados como $\mathbf{r}_{ \pm}^{\prime}=\mathbf{r}_{A}^{\prime} \pm \mathbf{r}_{D}^{\prime} / 2, \xi_{ \pm}=\xi_{A} \pm \xi_{D} / 2$ y $\mathbf{r}_{ \pm}=\mathbf{r}_{A} \pm \mathbf{r}_{D}$ /2. Los subíndices $A$ y $D$ no tienen significados particulares; su función es diferenciar la coordenada que señala la posición del punto medio entre una pareja de puntos, designada con el subíndice $A$, de la coordenada que especifica el vector de separación de dicha pareja, designada con el subíndice $D$. La Figura 1 indica que las parejas de puntos de cada plano están contenidas en regiones centradas en la coordenada con subíndice $A$, representadas por los círculos sombreados, las cuales se denominan "soportes estructurados de coherencia espacial” (Castañeda, 2014). Dichos soportes estructurados se caracterizan porque, (i) los valores de la correlación por fuera de ellos son nulos o despreciables; (ii) cada soporte estructurado contiene máximo una pareja de puntos con vector de separación específico, y (iii) los puntos de cada pareja son equidistantes del centro del soporte estructurado.

La preparación del experimento se describe mediante la función de correlación en el plano $\mathrm{M}$

$$
W_{M}\left(\xi_{+}, \xi_{-}\right)=\int_{S} d^{2} r_{A}^{\prime} \quad \mathbf{W}_{S M}\left(\mathbf{r}_{A}^{\prime} ; \xi_{+}, \xi_{-}\right),
$$

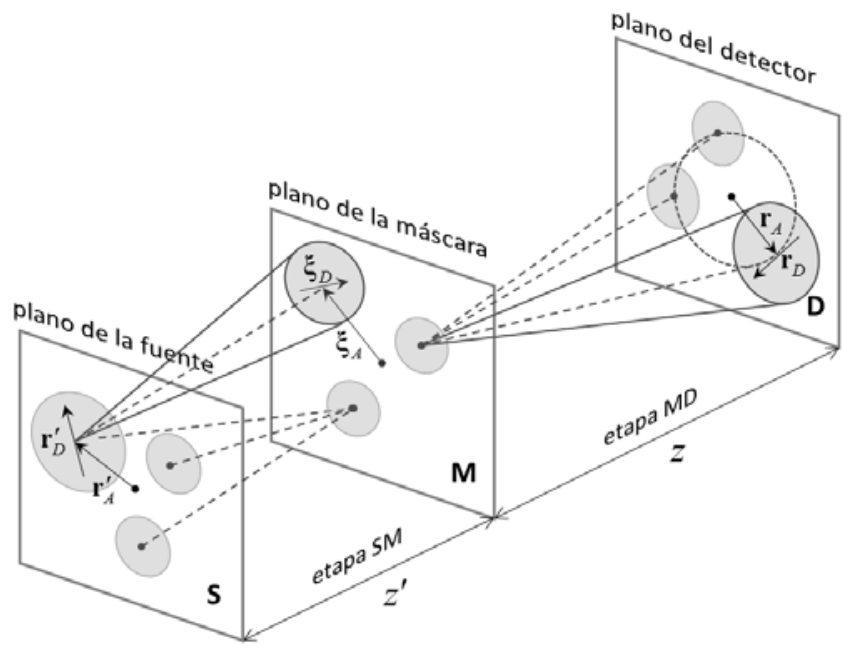

Figura 1. Diagrama conceptual de un montaje interferométrico experimental. Se incluyen las coordenadas reducidas de cada plano. Los círculos sombreados representan los soportes estructurados de coherencia espacial en el plano respectivo. Los conos de correlación están esquematizados en líneas sólidas.

cuyo integrando está dado por la expansión modal

$$
\mathbf{W}_{S M}\left(\mathbf{r}_{A}^{\prime} ; \xi_{+}, \xi_{-}\right)=\int_{S} d^{2} r_{D}^{\prime} W_{S}\left(\mathbf{r}_{+}^{\prime}, \mathbf{r}_{-}^{\prime}\right) \Phi_{S M}\left(\mathbf{r}_{+}^{\prime}, \mathbf{r}_{-}^{\prime} ; \xi_{+}, \xi_{-} ; k, z^{\prime}\right),
$$

con $W_{S}\left(\mathbf{r}_{+}^{\prime}, \mathbf{r}_{-}^{\prime}\right)$ como la correlación en el plano S y el kernel de la expansión dado por los modos no-paraxiales

$$
\begin{aligned}
& \Phi_{S M}\left(\mathbf{r}_{+}^{\prime}, \mathbf{r}_{-}^{\prime} ; \xi_{+}, \xi_{-} ; k, z^{\prime}\right)=\left(\frac{k}{4 \pi}\right)^{2} t_{S}\left(\mathbf{r}_{+}^{\prime}\right) t_{S}^{*}\left(\mathbf{r}_{-}^{\prime}\right) \\
& \times\left(\frac{z^{\prime}+\left|\mathbf{z}^{\prime}+\xi_{A}-\mathbf{r}_{A}^{\prime}+\left(\xi_{D}-\mathbf{r}_{D}^{\prime}\right) / 2\right|}{\left|\mathbf{z}^{\prime}+\xi_{A}-\mathbf{r}_{A}^{\prime}+\left(\xi_{D}-\mathbf{r}_{D}^{\prime}\right) / 2\right|^{2}}\right)\left(\frac{z^{\prime}+\left|\mathbf{z}^{\prime}+\xi_{A}-\mathbf{r}_{A}^{\prime}-\left(\xi_{D}-\mathbf{r}_{D}^{\prime}\right) / 2\right|}{\left|\mathbf{z}^{\prime}+\xi_{A}-\mathbf{r}_{A}^{\prime}-\left(\xi_{D}-\mathbf{r}_{D}^{\prime}\right) / 2\right|^{2}}\right),(1 \mathrm{c}), \\
& \times \exp \left(i k\left|\mathbf{z}^{\prime}+\xi_{A}-\mathbf{r}_{A}^{\prime}+\left(\xi_{D}-\mathbf{r}_{D}^{\prime}\right) / 2\right|-i k\left|\mathbf{z}^{\prime}+\xi_{A}-\mathbf{r}_{A}^{\prime}-\left(\xi_{D}-\mathbf{r}_{D}^{\prime}\right) / 2\right|\right)
\end{aligned}
$$

los cuales son funciones geométricas, determinísticas y escalares, definidas en el volumen de la etapa de preparación por las condiciones de frontera impuestas por su configuración. La función compleja $t_{S}\left(\mathbf{r}_{ \pm}^{\prime}\right)=\left|t_{S}\left(\mathbf{r}_{ \pm}^{\prime}\right)\right| \exp \left[i \phi_{S}\left(\mathbf{r}_{ \pm}^{\prime}\right)\right]$ se conoce como "función de transmisión de la fuente efectiva" de ondas o de partículas, ubicada en el plano S, la cual delimita la región del plano $\mathrm{S}$ en la que se emiten las ondas o las partículas. Además, $k=2 \pi / \lambda$, con $\lambda$ como la "métrica de escala espacial” (Castañeda, 2014), definida como la longitud a lo largo de la cual el propagador espacial del kernel evoluciona en $2 \pi$, en concordancia con las condiciones de frontera de la etapa. Por lo tanto, las expresiones $\lambda=2 \pi c / \omega, \lambda=h c / E$ y $\lambda=h / p$, que se obtienen vinculando las definiciones físicas de $k$ anteriormente mencionadas con esta definición geométrica, deben entenderse como condiciones de acople de las ondas y las partículas al montaje experimental. Así, las ondas cuya frecuencia $\omega$, los fotones cuya energía $E$ y las partículas masivas cuyo momento $p$ satisfagan tales condiciones de acople, se moverán en la etapa SM siguiendo la geometría de los modos con métrica de escala espacial $\lambda$. Es sorprendente cómo esta interpretación del parámetro $\lambda$, que difiere de su significado convencional de "longitud de 
onda", ayuda significativamente a remover el requerimiento de dualidad de la explicación del fenómeno de interferencia, como se discute más adelante. La ecuación (1a) formaliza la preparación del experimento en la etapa SM, describiendo, (i) la correlación de dos puntos en el plano $\mathrm{M}$ para $\xi_{D} \neq 0$, y (ii) la distribución de energía en el mismo plano, para $\xi_{D}=0$, esto es $S_{M}\left(\xi_{A}\right)=W_{M}\left(\xi_{A}, \xi_{A}\right)$.

Por su parte, el observable físico registrado por el detector en el plano D está dado por

$$
S_{D}\left(\mathbf{r}_{A}\right)=\int_{M} d^{2} \xi_{A} \quad \mathbf{W}_{M D}\left(\xi_{A} ; \mathbf{r}_{A}\right)
$$

cuyo integrando está dado por la expansión modal

$$
\mathbf{W}_{M D}\left(\boldsymbol{\xi}_{A} ; \mathbf{r}_{A}\right)=\int_{M} d^{2} \xi_{D} W_{M}\left(\xi_{+}, \xi_{-}\right) \Phi_{M D}\left(\xi_{+}, \boldsymbol{\xi}_{-} ; \mathbf{r}_{A} ; k, z\right),
$$

con el kernel de la expansión dado por las funciones geométricas, determinísticas y escalares que constituyen los modos no-paraxiales, definidos en el volumen de la etapa $\mathrm{MD}$ en concordancia con las condiciones de frontera de su configuración,

$$
\begin{aligned}
& \Phi_{M D}\left(\xi_{+}, \xi_{-} ; \mathbf{r}_{A} ; k, z\right)=\left(\frac{k}{4 \pi}\right)^{2} t_{M}\left(\xi_{+}\right) t_{M}^{*}\left(\xi_{-}\right) \\
& \times\left(\frac{z+\left|\mathbf{z}+\mathbf{r}_{A}-\xi_{A}-\xi_{D} / 2\right|}{\left|\mathbf{z}+\mathbf{r}_{A}-\xi_{A}-\xi_{D} / 2\right|^{2}}\right)\left(\frac{z+\left|\mathbf{z}+\mathbf{r}_{A}-\xi_{A}+\xi_{D} / 2\right|}{\left|\mathbf{z}+\mathbf{r}_{A}-\xi_{A}+\xi_{D} / 2\right|^{2}}\right) \\
& \times \exp \left(i k\left|\mathbf{z}+\mathbf{r}_{A}-\xi_{A}-\xi_{D} / 2\right|-i k\left|\mathbf{z}+\mathbf{r}_{A}-\xi_{A}+\xi_{D} / 2\right|\right)
\end{aligned}
$$

En esta expresión, $t_{M}\left(\xi_{ \pm}\right)=\left|t_{M}\left(\xi_{ \pm}\right)\right| \exp \left[i \phi_{M}\left(\xi_{ \pm}\right)\right]$es la "función de transmisión (compleja) de la máscara" que actúa como dispositivo de interferencia ubicado en el plano M. Más adelante será útil considerar la simetría hermítica de estos modos, $\Phi_{M D}\left(\boldsymbol{\xi}_{+}, \boldsymbol{\xi}_{-} ; \mathbf{r}_{A} ; k, z\right)=\Phi_{M D}^{*}\left(\boldsymbol{\xi}_{-}, \boldsymbol{\xi}_{+} ; \mathbf{r}_{A} ; k, z\right)$.

Un aspecto importante de las expansiones modales en las ecuaciones (1b) y (2b) es que la geometría de los modos no-paraxiales es independiente de la forma explícita de sus coeficientes. De hecho, la geometría de los modos solo puede cambiarse modificando la configuración de la etapa correspondiente. Consideremos ahora el significado físico de esas expresiones. Las cantidades $\mathbf{W}_{S M}\left(\mathbf{r}_{A}^{\prime} ; \boldsymbol{\xi}_{+}, \boldsymbol{\xi}_{-}\right) \mathrm{y}$ $\mathbf{W}_{M D}\left(\boldsymbol{\xi}_{A} ; \mathbf{r}_{A}\right)$ tienen unidades de energía, conferidas respectivamente por las correlaciones $W_{S}\left(\mathbf{r}_{+}^{\prime}, \mathbf{r}_{-}^{\prime}\right)$ y $W_{M}\left(\xi_{+}, \xi_{-}\right)$, y están definidas en todo el volumen de la etapa del montaje experimental señalizada en su subíndice. De manera más específica, describen distribuciones de energía sobre la geometría determinada por el kernel de la respectiva etapa. Los argumentos de $\mathbf{W}_{S M}\left(\mathbf{r}_{A}^{\prime} ; \xi_{+}, \xi_{-}\right)$indican que esta cantidad se distribuye sobre conos con vértices en cada punto $\mathbf{r}_{A}^{\prime}$ del plano S, y con bases sobre el soporte estructurado de coherencia espacial centrado en cada punto $\xi_{A}$ del plano $\mathrm{M}$. No obstante, el valor de la energía distribuida sobre dicha geometría se obtiene realizando la integral de la ecuación (1b) sobre el soporte estructurado centrado en el vértice del cono $\mathbf{r}_{A}^{\prime}$. Este cono, representado en líneas sólidas en la Figura 1, se denomina "cono de correlación”, ya que contribuye a la correlación sobre cada soporte estructurado en el plano M.
De forma similar, los argumentos de $\mathbf{W}_{M D}\left(\xi_{A} ; \mathbf{r}_{A}\right)$ indican que ésta se distribuye sobre conos con vértices en cada punto $\xi_{A}$ del plano M, y con bases sobre la región de puntos $\mathbf{r}_{A}$, correspondiente al área de detección en el plano D. El valor de la energía distribuida sobre dicha geometría se obtiene realizando la integral de la ecuación (2b) sobre el soporte estructurado centrado en el vértice del cono $\xi_{A}$. Este cono, representado en líneas punteadas en la Figura 1 , se denomina "cono de energía”, ya que su sección transversal determina el patrón de interferencia detectado en el plano D. Por lo tanto, las ecuaciones (1a) y (2a) indican, respectivamente, que, (i) la correlación sobre el soporte estructurado con centro en el punto $\xi_{A}$ del plano $\mathrm{M}$ es la sección transversal del cono resultante del solapamiento de los conos de correlación, con vértices en todos los puntos $\mathbf{r}_{A}^{\prime}$ del plano $\mathrm{S}$ y la misma base, correspondiente a dicho soporte estructurado; y (ii) la distribución de energía del patrón de interferencia en el plano D es la sección transversal del cono resultante del solapamiento de los conos de energía, con vértices en todos los puntos $\xi_{A}$ del plano $\mathrm{M}$ y la misma base, correspondiente al área de detección en el plano D.

\section{Grado de correlación y filtrado modal}

Las correlaciones en los planos $\mathrm{S}$ y $\mathrm{M}$ son funciones complejas que satisfacen las desigualdades de Schwarz (Mandel \& Wolf, 1995) $\left|W_{S}\left(\mathbf{r}_{+}^{\prime}, \mathbf{r}_{-}^{\prime}\right)\right|^{2} \leq S_{S}\left(\mathbf{r}_{+}^{\prime}\right) S_{S}\left(\mathbf{r}_{-}^{\prime}\right)$ y $\mid W_{M}\left(\xi_{+}, \xi_{-}\right)^{2} \leq S_{M}\left(\xi_{+}\right) S_{M}\left(\xi_{-}\right)$. Estas desigualdades pue-den escribirse como ecuaciones introduciendo, en sus miem-bros derechos, los factores $\left|\mu_{S}\left(\mathbf{r}_{+}^{\prime}, \mathbf{r}_{-}^{\prime}\right)\right|^{2}$ y $\left|\mu_{M}\left(\xi_{+}, \xi_{-}\right)\right|^{2}$, los cuales deben cumplir los siguientes requisitos:

$$
\begin{aligned}
& \text { - } \mu_{S}\left(\mathbf{r}_{+}^{\prime}, \mathbf{r}_{-}^{\prime}\right)=\left|\mu_{S}\left(\mathbf{r}_{+}^{\prime}, \mathbf{r}_{-}^{\prime}\right)\right| \exp \left[i \alpha_{S}\left(\mathbf{r}_{+}^{\prime}, \mathbf{r}_{-}^{\prime}\right)\right]_{\mathrm{y}} \\
& \mu_{M}\left(\xi_{+}, \xi_{-}\right)=\left|\mu_{M}\left(\xi_{+}, \xi_{-}\right)\right| \exp \left[i \alpha_{M}\left(\xi_{+}, \xi_{-}\right)\right] \text {, con } \\
& \alpha_{S}\left(\mathbf{r}_{+}^{\prime}, \mathbf{r}_{-}^{\prime}\right)=\arg \left[W_{S}\left(\mathbf{r}_{+}^{\prime}, \mathbf{r}_{-}^{\prime}\right)\right]_{\mathrm{y}} \alpha_{M}\left(\xi_{+}, \xi_{-}\right)=\arg \left[W_{M}\left(\xi_{+}, \xi_{-}\right)\right] \text {; } \\
& \bullet 0 \leq\left|\mu_{S}\left(\mathbf{r}_{+}^{\prime}, \mathbf{r}_{-}^{\prime}\right)\right| \leq 1 \text { y } ; 0 \leq\left|\mu_{M}\left(\xi_{+}, \xi_{-}\right)\right| \leq 1 \text {; } \\
& \text { - } \mu_{S}\left(\mathbf{r}_{A}^{\prime}, \mathbf{r}_{A}^{\prime}\right)=1 \text { y } \alpha_{S}\left(\mathbf{r}_{A}^{\prime}, \mathbf{r}_{A}^{\prime}\right)=0 \text {, y, de forma similar, } \\
& \mu_{M}\left(\xi_{A}, \xi_{A}\right)=1 \text { y } \alpha_{M}\left(\xi_{A}, \xi_{A}\right)=0, \mathrm{y} \\
& \text { - } \mu_{S}\left(\mathbf{r}_{+}^{\prime}, \mathbf{r}_{-}^{\prime}\right)=\mu_{S}^{*}\left(\mathbf{r}_{-}^{\prime}, \mathbf{r}_{+}^{\prime}\right) \text { y } \mu_{M}\left(\xi_{+}, \boldsymbol{\xi}_{-}\right)=\mu_{M}^{*}\left(\xi_{-}, \xi_{+}\right) \text {. }
\end{aligned}
$$

$\mu_{S}\left(\mathbf{r}_{+}^{\prime}, \mathbf{r}_{-}^{\prime}\right)$ y $\mu_{M}\left(\xi_{+}, \xi_{-}\right)$se conocen como "grados complejos de correlación (o de coherencia) espacial”. Así, las correlaciones en los planos $\mathrm{S}$ y $\mathrm{M}$ pueden expresarse como $W_{S}\left(\mathbf{r}_{+}^{\prime}, \mathbf{r}_{-}^{\prime}\right)=\mu_{S}\left(\mathbf{r}_{+}^{\prime}, \mathbf{r}_{-}^{\prime}\right) \sqrt{S_{S}\left(\mathbf{r}_{+}^{\prime}\right)} \sqrt{S_{S}\left(\mathbf{r}_{-}^{\prime}\right)}$ y $W_{M}\left(\boldsymbol{\xi}_{+}, \boldsymbol{\xi}_{-}\right)=\mu_{M}\left(\boldsymbol{\xi}_{+}, \boldsymbol{\xi}_{-}\right)$ $\sqrt{S_{M}\left(\xi_{+}\right)} \sqrt{S_{M}\left(\xi_{-}\right)}$. Estas definiciones indican que, (i) el carácter estadístico de las correlaciones deviene de las fluctuaciones en las energías $S_{S}\left(\mathbf{r}_{ \pm}^{\prime}\right)_{\text {y }} S_{M}\left(\boldsymbol{\xi}_{ \pm}\right)$de las ondas y de las partículas, y (ii) los soportes de $\mu_{S}\left(\mathbf{r}_{+}^{\prime}, \mathbf{r}_{-}^{\prime}\right)$ y $\mu_{M}\left(\boldsymbol{\xi}_{+}, \boldsymbol{\xi}_{-}\right)$, con centros en los puntos $\mathbf{r}_{A}^{\prime}$ del plano S y $\xi_{A}$ del plano $\mathrm{M}$, respectivamente, son los soportes estructurados de coherencia espacial centrados en dichos puntos.

Luego de reemplazar las anteriores definiciones de las correlaciones en las ecuaciones (1b) y (2b), se observa que $\mu_{S}\left(\mathbf{r}_{+}^{\prime}, \mathbf{r}_{-}^{\prime}\right)$ y $\mu_{M}\left(\xi_{+}, \xi_{-}\right)$se comportan como filtros modales en las respectivas expansiones. En efecto, los grados de correlación suprimen los modos asociados a parejas de 
puntos cuyas separaciones son mayores que el tamaño de sus soportes, y sopesan los modos de las parejas incluidas en sus soportes con valores en el intervalo $[0,1]$. Teniendo en cuenta que dichas expansiones modales describen completamente los experimentos de interferencia, se concluye que la interferencia es consecuencia de la filtración realizada por el grado de correlación sobre los modos no-paraxiales definidos en el volumen de cada etapa del montaje experimental. Por lo tanto, el conjunto máximo de modos involucrados en la producción de patrones de interferencia, tanto de ondas como de partículas, es determinado por el montaje experimental bajo la condición $\mu_{S}\left(\mathbf{r}_{+}^{\prime}, \mathbf{r}_{-}^{\prime}\right)=1$ y $\mu_{M}\left(\xi_{+}, \xi_{-}\right)$ $=1$, en tanto que el conjunto mínimo se obtiene bajo la condición $\mu_{S}\left(\mathbf{r}_{+}^{\prime}, \mathbf{r}_{-}^{\prime}\right)=0$ para todo $\mathbf{r}_{D}^{\prime} \neq 0$ y $\mu_{M}\left(\xi_{+}, \xi_{-}\right)=0$ y para todo $\xi_{D} \neq 0$.

\section{Una reinterpretación del teorema de Van Cittert- Zernike}

Los experimentos de interferencia en los que la emisión de ondas o de partículas por la fuente efectiva es no-correlacionada revisten especial interés. Esto ocurre, por ejemplo, cuando la fuente es térmica, como en el experimento original de Young realizado con luz solar (Born \& Wolf, 1993), en los experimentos con electrones singulares producidos por termo-ionización (Frabboni, et al., 2012; Matteucci, et al., 2013), o en los que se emplean moléculas individuales por sublimación (Nairz, et al., 2003; Juffmann, et al., 2012). En estos experimentos se cumple que $\mu_{S}\left(\mathbf{r}_{+}^{\prime}, \mathbf{r}_{-}^{\prime}\right)=0$ para todo $\mathbf{r}_{D}^{\prime} \neq 0$, de manera que la ecuación (1b) conduce a

$$
\mathbf{W}_{S M}\left(\mathbf{r}_{A}^{\prime} ; \boldsymbol{\xi}_{+}, \boldsymbol{\xi}_{-}\right)=S_{S}\left(\mathbf{r}_{A}^{\prime}\right) \Phi_{S M}\left(\mathbf{r}_{A}^{\prime} ; \boldsymbol{\xi}_{+}, \xi_{-} ; k, z^{\prime}\right),
$$

y, por lo tanto, la correlación preparada en el plano M según la ecuación (1a) toma la forma

$$
W_{M}\left(\xi_{+}, \xi_{-}\right)=\int_{S} d^{2} r_{A}^{\prime} S_{S}\left(\mathbf{r}_{A}^{\prime}\right) \Phi_{S M}\left(\mathbf{r}_{A}^{\prime} ; \xi_{+}, \xi_{-} ; k, z^{\prime}\right) .
$$

La ecuación (3a) indica que la energía de cada emisión local de ondas o de partículas en el plano S, $S_{S}\left(\mathbf{r}_{A}^{\prime}\right)$, contribuirá a la correlación sobre el soporte estructurado centrado en cada punto $\xi_{A}$ del plano $\mathrm{M}$, debido al cono de correlación determinado por el modo no-paraxial $\Phi_{S M}\left(\mathbf{r}_{A}^{\prime} ; \xi_{+}, \xi_{-} ; k, z^{\prime}\right)$ . Según la ecuación (3b), la sección transversal del cono resultante del solapamiento de todos los conos de correlación establecerá la correlación $W_{M}\left(\xi_{+}, \xi_{-}\right)$sobre dicho soporte estructurado, a pesar de que las emisiones locales de la fuente efectiva sean no-correlacionadas. En otras palabras, la correlación en el plano M se debe a la geometría del kernel modal en la etapa SM del montaje experimental.

En óptica, la ecuación (3b) se conoce como teorema de Van Cittert-Zernike (no-paraxial) (Castañeda, 2014), y se interpreta como la predicción de que las ondas emitidas en procesos no-correlacionados ganan correlación espacial solo a causa de su propagación. Sin embargo, esta interpretación no es aplicable a la preparación de experimentos de interferencia con partículas singulares, puesto que, aunque la correlación de sus funciones de onda cuántica pueda calcularse, esta no tiene un significado físico preciso que pueda respaldar la hipótesis de ganancia de correlación como atributo físico de las partículas individuales debido a su movimiento. En cambio la interpretación geométrica de la ecuación (3b) es independiente de tales atributos físicos asignados a las ondas o a las partículas, al tiempo que mantiene la precisión predictiva de los resultados experimentales, como se ilustrará más adelante.

Asimismo, la ecuación (1a) es congruente con esta interpretación, lo cual indica que los cambios geométricos de los conos de correlación se deben a la inclusión de más modos no-paraxiales en el kernel cuando las emisiones locales de ondas o de partículas son parcial o totalmente correlacionadas. En efecto, las variaciones de los conos de correlación debidas a esta causa se evidencian escribiendo la ecuación (1b) como sigue:

$$
\begin{aligned}
& \mathbf{W}_{S M}\left(\mathbf{r}_{A}^{\prime} ; \boldsymbol{\xi}_{+}, \boldsymbol{\xi}_{-}\right)=S_{S}\left(\mathbf{r}_{A}^{\prime}\right) \Phi_{S M}\left(\mathbf{r}_{A}^{\prime} ; \boldsymbol{\xi}_{+}, \boldsymbol{\xi}_{-} ; k, z^{\prime}\right) \\
& +\int_{\substack{S \\
\mathbf{r}_{D}^{\prime} \neq 0}} d^{2} r_{D}^{\prime} \mu_{S}\left(\mathbf{r}_{+}^{\prime}, \mathbf{r}_{-}^{\prime}\right) \sqrt{S_{S}\left(\mathbf{r}_{+}^{\prime}\right)} \sqrt{S_{S}\left(\mathbf{r}_{-}^{\prime}\right)} \Phi_{S M}\left(\mathbf{r}_{+}^{\prime}, \mathbf{r}_{-}^{\prime} ; \xi_{+}, \xi_{-} ; k, z^{\prime}\right),
\end{aligned}
$$

donde el primer término describe la contribución de las emisiones no-correlacionadas en el plano $\mathrm{S}$ a la correlación en el plano $\mathrm{M}$, mientras que el segundo describe las contribuciones aportadas por las emisiones con un cierto grado de correlación.

\section{Una nueva ley general de interferencia}

La interferencia de ondas y de partículas se formaliza a través de una ley matemática que en óptica lleva el nombre de ley general de interferencia (Born \& Wolf, 1993), la cual se refiere al experimento de Young, ya sea con ondas (Born \& Wolf, 1993), o con partículas (Zeilinger, et al., 1988; Matteucci, et al, 2013), cuyo desarrollo se presenta en la sección B de la información suplementaria.

La preparación del experimento en la etapa SM consiste en determinar la correlación y la distribución de energía en el plano M, las cuales se obtienen a partir de las ecuaciones (1a) y (B2), así:

$$
\begin{aligned}
& W_{M}\left(\xi_{+}, \xi_{-}\right)=\left(\frac{k}{4 \pi}\right)^{2} S_{S}(0) \\
& \quad\left(\frac{z^{\prime}+\left|\mathbf{z}^{\prime}+\xi_{A}+\xi_{D} / 2\right|}{\left|\mathbf{z}^{\prime}+\xi_{A}+\xi_{D} / 2\right|^{2}}\right)\left(\frac{z^{\prime}+\left|\mathbf{z}^{\prime}+\xi_{A}-\xi_{D} / 2\right|}{\left|\mathbf{z}^{\prime}+\xi_{A}-\xi_{D} / 2\right|^{2}}\right), \\
& \quad \times \exp \left(i k\left|\mathbf{z}^{\prime}+\xi_{A}+\xi_{D} / 2\right|-i k\left|\mathbf{z}^{\prime}+\xi_{A}-\xi_{D} / 2\right|\right) \\
& \mathrm{y} \quad S_{M}\left(\xi_{A}\right)=W_{M}\left(\xi_{A}, \xi_{A}\right)=\left(\frac{k}{4 \pi}\right)^{2} S_{S}(0)\left(\frac{z^{\prime}+\left|\mathbf{z}^{\prime}+\xi_{A}\right|}{\left|\mathbf{z}^{\prime}+\xi_{A}\right|^{2}}\right)^{2} .
\end{aligned}
$$

Ahora bien, la máscara en el plano $M$ tendrá solamente un par de agujeros circulares idénticos y de tamaño arbitrariamente pequeño. Estos agujeros, conocidos como pinholes, se ubican de manera que el punto medio entre ellos esté en $\xi_{A}=0$ y su vector de separación sea $\xi_{D}=\mathbf{b}$. Así, la pareja de pinholes estará correlacionada solo en el caso en que quede inscrita en el soporte estructurado con diámetro $\sigma>b$ y centro en $\xi_{A}=0$. De acuerdo con la ecuación (5a), su correlación es 


$$
\begin{gathered}
W_{M}(\mathbf{b})=\left(\frac{k}{4 \pi}\right)^{2} S_{S}(0)\left(\frac{z^{\prime}+\left|\mathbf{z}^{\prime}+\mathbf{b} / 2\right|}{\left|\mathbf{z}^{\prime}+\mathbf{b} / 2\right|^{2}}\right)\left(\frac{z^{\prime}+\left|\mathbf{z}^{\prime}-\mathbf{b} / 2\right|}{\left|\mathbf{z}^{\prime}-\mathbf{b} / 2\right|^{2}}\right) . \\
\quad \exp \left(i k\left|\mathbf{z}^{\prime}+\mathbf{b} / 2\right|-i k\left|\mathbf{z}^{\prime}-\mathbf{b} / 2\right|\right)
\end{gathered}
$$

Por su parte, la energía de las ondas o las partículas que atraviesan los pinholes de la máscara $\mathrm{M}$ se obtiene evaluando la ecuación (5b) para $\xi_{A}= \pm \mathbf{b} / 2$, esto es

$$
S_{M}( \pm \mathbf{b} / 2)=\left(\frac{k}{4 \pi}\right)^{2} S_{S}(0)\left(\frac{z^{\prime}+\left|\mathbf{z}^{\prime} \pm \mathbf{b} / 2\right|}{\left|\mathbf{z}^{\prime} \pm \mathbf{b} / 2\right|^{2}}\right)^{2} .
$$

Así, las ondas o las partículas atravesarán los pinholes con la misma energía siempre que el área iluminada tenga un diámetro $\varepsilon>b$, incluso si los eventos de emisión son singulares. Además, como la fuente efectiva es también un pinhole, se tiene que $\sigma>\varepsilon>b$, es decir, las ondas o las partículas atravesarán pinholes correlacionados en el plano $\mathrm{M}$, incluso cuando solo uno de ellos es atravesado por una partícula singular. En otras palabras, la correlación no será una característica no-local de las ondas o de las partículas que se mueven en el interferómetro, sino un atributo extendido de la configuración del montaje experimental. Las ecuaciones (6) representan, entonces, la preparación del experimento de interferencia de Young.

Consideremos ahora las ecuaciones (2a) y (5b), las cuales permiten expresar la distribución de energía del patrón de interferencia en el plano D como $S_{D}\left(\mathbf{r}_{A}\right)=S_{D}^{(+)}\left(\mathbf{r}_{A}\right)+$ $S_{D}^{(-)}\left(\mathbf{r}_{A}\right)$, con

$$
S_{D}^{( \pm)}\left(\mathbf{r}_{A}\right)=S_{R}^{( \pm)}\left(\mathbf{r}_{A}\right)+S_{V}\left(\mathbf{r}_{A}\right)
$$

como las distribuciones de energía sobre las secciones transversales de los conos $\mathbf{W}_{M D}^{( \pm)}\left(\boldsymbol{\xi}_{A} ; \mathbf{r}_{A}\right)$, los cuales se muestran en la Figura 2, tanto en la región cercana al plano M como en el campo lejano. Los términos de la ecuación (7) son

$$
S_{R}^{( \pm)}\left(\mathbf{r}_{A}\right)=\left(\frac{k}{4 \pi}\right)^{2} S_{M}( \pm \mathbf{b} / 2)\left(\frac{z+\left|\mathbf{z}+\mathbf{r}_{A} \pm \mathbf{b} / 2\right|}{\left|\mathbf{z}+\mathbf{r}_{A} \pm \mathbf{b} / 2\right|^{2}}\right)^{2}
$$

$\mathrm{y}$

$$
\begin{aligned}
& S_{V}\left(\mathbf{r}_{A}\right)=\left(\frac{k}{4 \pi}\right)^{2}\left|\mu_{M}(\mathbf{b})\right| \sqrt{S_{M}(\mathbf{b} / 2)} \sqrt{S_{M}(-\mathbf{b} / 2)} \\
& \times\left(\frac{z+\left|\mathbf{z}+\mathbf{r}_{A}-\mathbf{b} / 2\right|}{\left|\mathbf{z}+\mathbf{r}_{A}-\mathbf{b} / 2\right|^{2}}\right)\left(\frac{z+\left|\mathbf{z}+\mathbf{r}_{A}+\mathbf{b} / 2\right|}{\left|\mathbf{z}+\mathbf{r}_{A}+\mathbf{b} / 2\right|^{2}}\right) . \\
& \cos \left(k\left|\mathbf{z}+\mathbf{r}_{A}-\mathbf{b} / 2\right|-k\left|\mathbf{z}+\mathbf{r}_{A}+\mathbf{b} / 2\right|+\alpha_{M}(\mathbf{b})\right)
\end{aligned}
$$

La similitud geométrica de los conos correspondientes en la Figura 2 es notable, incluso considerando su marcada diferencia de escala. La condición $S_{D}^{( \pm)}\left(\mathbf{r}_{A}\right) \geq 0$ se cumple en todos los puntos de la región delimitada por líneas punteadas en los grafos (a1), (a3), (b1) y (b3), ya que en esta región la magnitud del valor negativo del término cosenoidal de la ecuación (7) en un punto dado no supera el valor positivo del primer término de dicha ecuación en el mismo punto. Tal condición se extiende a toda la sección transversal del cono a distancias de campo lejanas del plano $\mathrm{M}$, como se ilustra en los grafos (a2), (a4), (b2) y (b4).

Es importante señalar que si se retira el cono modulador, la región en la que se cumple la condición $S_{D}^{( \pm)}\left(\mathbf{r}_{A}\right) \geq 0$ corresponderá al volumen del respectivo cono real. Se concluye, entonces, que $S_{D}^{( \pm)}\left(\mathbf{r}_{A}\right) \geq 0$ es una condición física para el movimiento de ondas y partículas en la etapa MD del montaje experimental. Tal condición implica que los puntos en los cuales $S_{D}^{( \pm)}\left(\mathbf{r}_{A}\right)<0$ constituyen zonas prohibidas para dicho movimiento.

La imagen (c1) de la Figura 2 es el resultado del solapamiento de los conos en las imágenes (a1) y (b1). Similarmente, la imagen (c2) resulta de superponer (a2) y (b2), y el mismo procedimiento se aplica para las imágenes (c3) y (c4). Los perfiles de las secciones transversales de los grafos en la fila (c) de la Figura 2 describen los patrones de interferencia del experimento de Young, registrados por el detector en el plano D. Estos patrones cumplen la condición $S_{D}\left(\mathbf{r}_{A}\right) \geq 0$ en el volumen completo de la etapa MD.

\section{Una descripción alternativa del fenómeno de interferencia}

De acuerdo con la descripción anterior del experimento de Young, la interferencia, tanto de ondas como de partículas, puede explicarse de la siguiente manera.

(i) La fuente efectiva tiene eventos locales de emisión de ondas o partículas que se mueven hacia el plano $\mathrm{M}$, donde está ubicada la máscara con dos pinholes. Las partículas singulares solo pueden cruzar uno de ellos, pero las ondas pueden establecer perturbaciones en ambos pinholes al tiempo. A pesar de esta simultaneidad, el hecho de que los conos de energía asociados a los pinholes no puedan modularse mutuamente caracteriza el cruce de la energía ondulatoria por ellos como dos eventos locales separados. El arribo de las ondas y de las partículas al plano D es registrado localmente por el detector en ese plano.

(ii) El carácter no-local o extendido del fenómeno de interferencia se debe a la geometría de los conos modulados, determinados por los modos no-paraxiales de los kernels de cada etapa del montaje experimental. Como en este modelo interpretativo la determinación del cono modulador es independiente de las ondas y las partículas que se mueven en el interferómetro, el principio de superposición de ondas no es aplicable.

(iii) Si ambos pinholes están inscritos dentro del mismo cono de correlación y permanecen abiertos durante el lapso entre un evento de emisión y su correspondiente evento de detección, la onda o la partícula emitidas (incluso individualmente) se moverán a través del cono modulado completo en la etapa SM. En consecuencia, un patrón de franjas de interferencia se producirá luego de un número suficientemente alto de eventos de detección.

(iv) Si ambos pinholes no pueden inscribirse en el mismo cono de correlación o uno de los pinholes es bloqueado, el cono modulador desaparece en el volumen de la etapa MD. Así, las perturbaciones ondulatorias o las partículas singulares que cruzan los pinholes se moverán dentro del cono real lorentziano resultante en dicha etapa y no producirán patrones de franjas de interferencia en el plano D. 
$\lambda=632 \mathrm{~nm}$

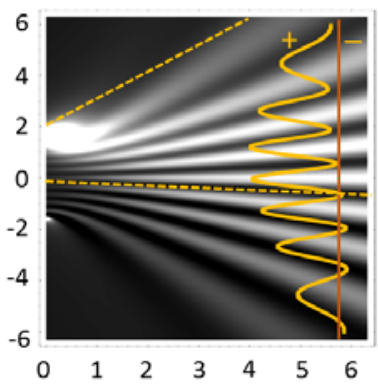

a1

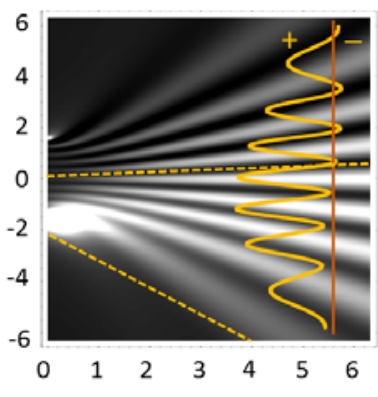

b1

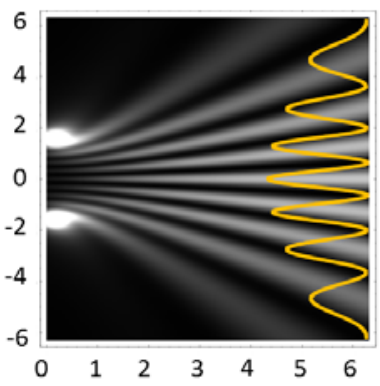

c1

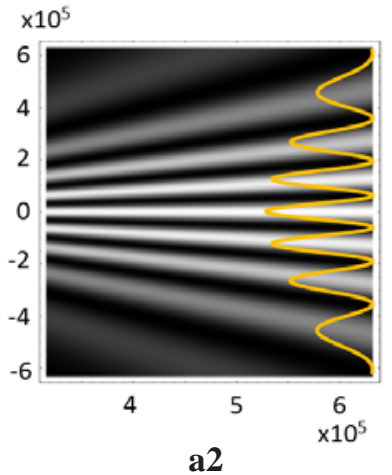

$\times 10^{5}$

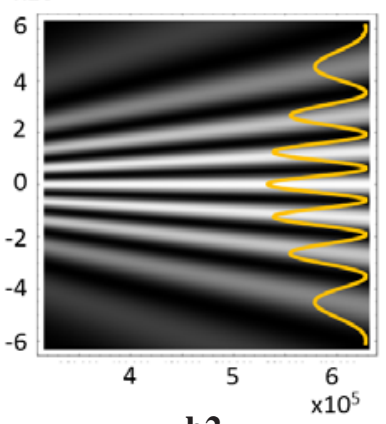

b2

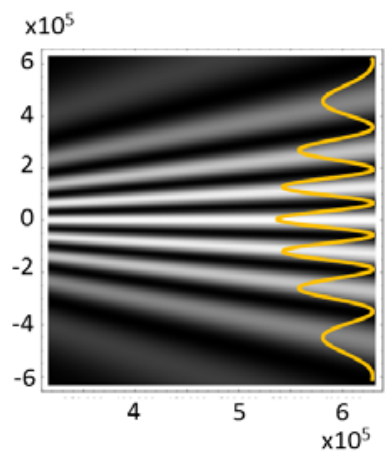

c2

$\lambda=4 \mathrm{pm}$

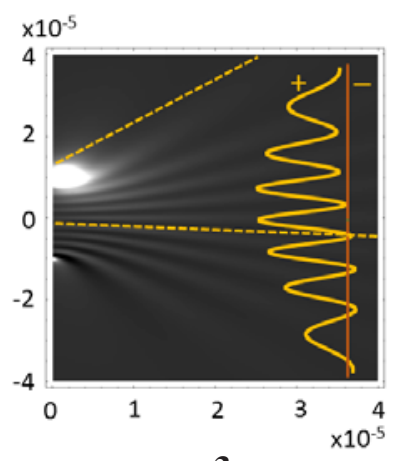

a3

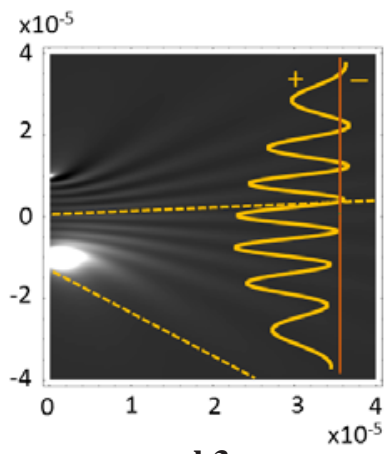

b3

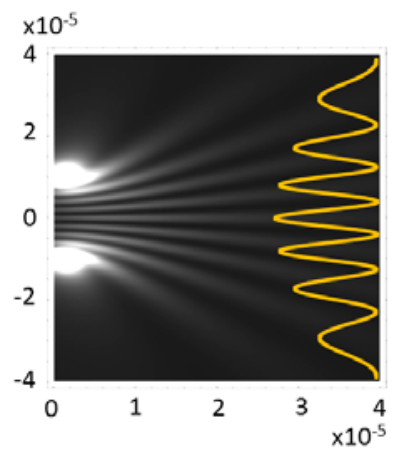

c3
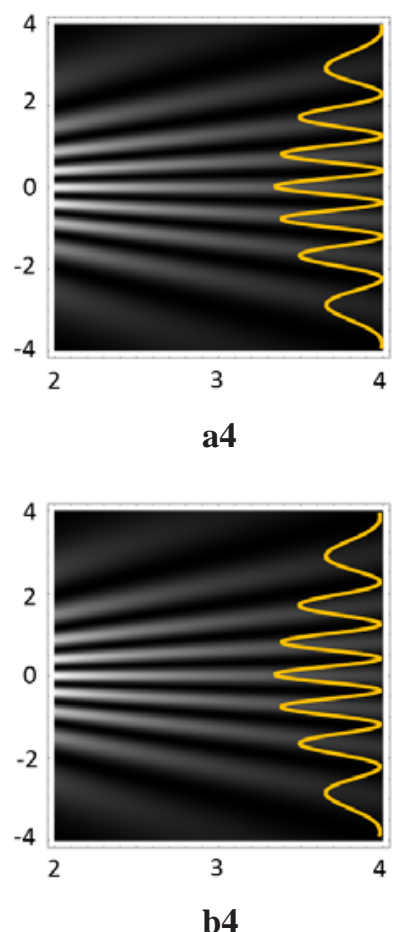

b4

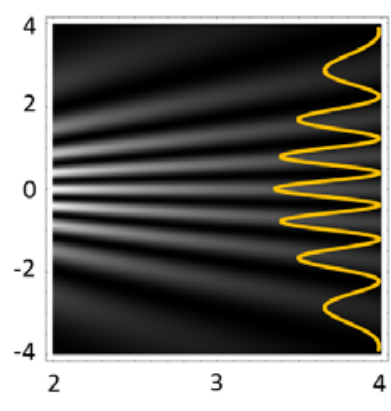

c4

Figura 2. Conos $W_{M D}^{(+)}\left(\xi_{A}, \mathbf{r}_{A}\right)$ en la fila superior, $W_{M D}^{(-)}\left(\boldsymbol{\xi}_{A}, \mathbf{r}_{A}\right)$ en la fila media y $W_{M D}\left(\boldsymbol{\xi}_{A}, \mathbf{r}_{A}\right)$ en la fila inferior. Las imágenes en las columnas 1 y 3 muestran las porciones de los conos cerca al plano $M$, indicando que sus vértices se ubican en $\xi_{A}= \pm$ b/2 y que los conos solo toman valores positivos en las regiones delimitadas por las líneas punteadas. Las imágenes de las columnas 2 y 4 muestran las porciones en campo lejano. Los grafos en las columnas 1 y 2 corresponden a interferencia de ondas, y fueron resaltados por motivos de presentación, en tanto que los de las columnas 3 y 4 corresponden a la interferencia de partículas singulares y muestran el decaimiento debido a la ley $1 / z^{2}$, que también se cumple en la interferencia con ondas. Para ambos casos de interferencia se empleó $\mu(b)=1 \mathrm{y} b=5 \lambda$. Las unidades de los ejes en todos los grafos son $\mu \mathrm{m}$. El eje horizontal es $z$ y los ejes verticales son $\xi_{A}$ para $z=0$ y $\mathrm{r}_{A}$ para $z>0$. Los perfiles verticales son las secciones transversales de los conos a lo largo del extremo derecho vertical de las imágenes. El aumento paulatino de la frecuencia espacial de las franjas hacia el centro del patrón se debe a la no linealidad del argumento del propagador espacial en el kernel modal (Castañeda, 2014).

(v) De acuerdo con (iii) y (iv), la noción de dualidad es innecesaria en este modelo interpretativo para explicar la construcción de patrones de interferencia por partículas singulares. Este aspecto invita a revisar el papel de ideas ampliamente aceptadas como el colapso de la función de onda, la deslocalización de partículas y la auto-interferencia.

(vi) La estadística del proceso de detección depende de la estadística del proceso de emisión y de la probabilidad de que un evento de emisión en la fuente efectiva realice un evento de cruce de pinhole en la máscara del plano M. Por lo tanto, se requiere un gran número de eventos de emisión para llenar la geometría del cono modulado en la etapa MD y producir el patrón de franjas de interferencia en el plano D.

La explicación anterior sugiere que la ecuación (7) tiene carácter de ley natural en la región donde $S_{D}^{( \pm)}\left(\mathbf{r}_{A}\right)$ es un observable físico, esto es, bajo la condición $S_{D}^{( \pm)}\left(\mathbf{r}_{A}\right) \geq 0$. 
En efecto, ella predice con total precisión el movimiento de ondas y partículas en la etapa MD de cualquier interferómetro de Young. Esta afirmación se ve validada por:

(i) la detección de partículas, cuadro por cuadro, en experimentos de interferencia con partículas singulares (Nairz, et al., 2003; Juffmann, et al., 2009; Matteucci, et al., 2013). Cada cuadro registra, como máximo, un único evento de detección de energía $S_{D}^{( \pm)}\left(\mathbf{r}_{A}\right)$, de manera que el patrón de interferencia se obtiene mediante la adición de dichos cuadros, esto es, $S_{D}^{(+)}\left(\mathbf{r}_{A}\right)+S_{D}^{(-)}\left(\mathbf{r}_{A}\right)$, y por

(ii) la detección de patrones de interferencia de ondas con distribución de energía $S_{D}^{(+)}\left(\mathbf{r}_{A}\right)+S_{D}^{(-)}\left(\mathbf{r}_{A}\right)$ en un único cuadro (Born \& Wolf, 1993).

Por estas razones, la ecuación (7) constituye la ley general de interferencia en el contexto de la nueva interpretación, cuya aplicación opera para ondas clásicas y partículas de materia. Esta ley es más fundamental que la ley convencional de interferencia óptica (Born \& Wolf, 1993; Mandel \& Wolf, 1995), solo formalizada por la suma $S_{D}^{(+)}\left(\mathbf{r}_{A}\right)+S_{D}^{(-)}\left(\mathbf{r}_{A}\right)$.

\section{Potencial geométrico y principio de interferencia}

Es claro que $\left|\mu_{M}(\mathbf{b})\right|$ disminuye si $b \rightarrow \sigma$, con lo cual el efecto del cono modulador sobre los conos reales en la etapa MD se debilita. En consecuencia, el contraste de las franjas se reduce en el cono modulado y, por consiguiente, en el patrón de interferencia en el plano D. Este comportamiento puede verificarse experimentalmente con solo aumentar el diámetro $a$ de la fuente efectiva, sin cambiar sus propiedades físicas y estadísticas. Ello indica que los significados físicos de los dos términos de la ecuación (7), y por ende de los conos real y modulador, son muy diferentes.

El primer término, definido en la ecuación (8a), es una función real y definida positivamente, que determina el cono real, de sección transversal lorentziana, cuya geometría es independiente de la correlación en el plano M. Este término corresponde a la energía de la perturbación ondulatoria o de la partícula que cruza uno de los pinholes, la cual es un observable físico. El segundo término, definido en la ecuación (8b), es una función determinada esencialmente por la configuración de la etapa $\mathrm{MD}$, y toma valores positivos y negativos, y, por lo tanto, no puede ser un observable físico, a pesar de sus unidades de energía. Este término especifica la geometría del cono modulador, y su peso relativo está dado principalmente por la correlación en el plano $\mathrm{M}$, cuyo significado, también geométrico, está ligado a la configuración de la etapa de preparación SM. Por tal razón, denominamos dicho término como "potencial geométrico" (Castañeda, 2017 a), ya que la modulación que introduce sobre el cono real actúa sobre el movimiento de las ondas y las partículas en la etapa MD, dirigiéndolo hacia las franjas brillantes de los grafos de la Figura 2.

Debe enfatizarse que el potencial geométrico no está asociado a un agente físico representable mediante un término explícito en la ecuación de Helmholtz, como ocurre, por ejemplo, con el potencial coulombiano de la ecuación de Schrödinger para el electrón orbital de un átomo. No obstante, el potencial geométrico está implícito en la expansión modal que determina la correlación en el fenómeno de interferencia, y que implica dos ecuaciones acopladas de Helmholtz.

El análisis anterior sugiere que la ecuación (7) representa la interacción entre la perturbación ondulatoria o la partícula individual que atraviesa uno de los pinholes y el arreglo experimental del interferómetro de Young. Para caracterizar apropiadamente esta interacción, se postula la existencia de (i) un "emisor puntual real", ubicado en el vértice de cada cono real, el cual da cuenta de la energía de la perturbación ondulatoria o de la partícula en el pinhole en dicho vértice; y (ii) un "emisor puntual virtual”, ubicado en el punto medio entre los pinholes, el cual da cuenta de la energía del potencial geométrico en la etapa MD (ver Figura S1) (Castañeda, et al., 2016 a; Castañeda, 2017 a). Así, la nueva ley general de interferencia, dada por la ecuación (7), establece la energía de la interacción no-local entre un emisor puntual real y un emisor puntual virtual, interacción que constituye el nuevo "principio generalizado de interferencia” para ondas y partículas (Castañeda, 2017 a). Sus aspectos más destacables son los siguientes.

(i) Las interacciones descritas por $S_{D}^{(+)}\left(\mathbf{r}_{A}\right)$ y $S_{D}^{(-)}\left(\mathbf{r}_{A}\right)$ ocurren de manera separada, incluso en el caso de perturbaciones ondulatorias que cruzan ambos pinholes al tiempo. El patrón de interferencia resultará, entonces, de la adición de todas las interacciones realizadas.

(ii) Para que una interacción ocurra, ambos pinholes tienen que estar abiertos durante el evento de cruce de la máscara en el plano $\mathrm{M}$, incluso si el experimento se hace con partículas singulares. El bloqueo de uno de los pinholes anula el grado de correlación $\mu$ (b), eliminando al emisor puntual virtual, lo cual destruye el potencial geométrico. Esto confirma, una vez más, el estrecho vínculo causal entre el montaje experimental y el potencial geométrico.

(iii) La interacción entre emisores puntuales reales y virtuales es conservativa. Previamente se ha establecido que $\int d^{2} r_{A} \Phi_{M D}\left(\xi_{A} ; \mathbf{r}_{A} ; k, z\right)=1$ y $\int d^{2} r_{A} \Phi_{M D}\left(\xi_{+}, \xi_{-} ; \mathbf{r}_{A} ; k, z\right)=0$ (Caskañeda, 2014; Castañeda, $D 2017$ a) y, por lo tanto, de las ecuaciones (2) se sigue que $\int_{D} d^{2} r_{A} S_{D}\left(\mathbf{r}_{A}\right)=\int_{M} d^{2} \xi_{A} \int_{D} d^{2} r_{A} \mathbf{w}_{M D}\left(\xi_{A} ; \mathbf{r}_{A}\right)=$ $\int d^{2} \xi_{A} S_{M}\left(\xi_{A}\right)$.

\section{Interferencia con fuentes extendidas y rejillas}

Consideremos ahora los experimentos preparados con fuentes efectivas extendidas y realizados con rejillas de pinholes (arreglos lineales regularmente espaciados). Los conos de correlación producidos por fuentes efectivas extendidas en la etapa SM son de apertura angular más estrecha y de geometría diferente a la de los conos de correlación lorentzianos, producidos por un único pinhole. En consecuencia, las parejas de puntos en el plano $\mathrm{M}$ son, en general, parcialmente correlacionadas, y se necesitan varios conos de correlación para cubrir todas las parejas de puntos incluidas en la rejilla. Esto significa que, para caracterizar completamente este 
tipo de experimentos, se requieren conjuntos discretos de emisores puntuales reales (un emisor por cada pinhole de la rejilla) y de emisores puntuales virtuales (un emisor por cada cono de correlación requerido) distribuidos en el plano M, así como sus correspondientes modos no-paraxiales en todo el volumen de la etapa MD.

Una novedosa herramienta, denominada "espectro de clases de emisores puntuales” (Castañeda \& Muñoz, 2016), se ha diseñado específicamente para esta caracterización. El espectro de clases permite construir el mapa completo de las interacciones entre los emisores puntuales reales y virtuales que se presentan en un experimento específico. Dicho espectro se representa como un grafo de puntos distribuidos en un plano de ejes cartesianos $\left(\boldsymbol{\xi}_{A}, \boldsymbol{\xi}_{D}\right)$, donde la abscisa $\boldsymbol{\xi}_{A}$ especifica la posición del emisor puntual sobre el plano $\mathrm{M}$, del cual el punto considerado es componente, mientras que la ordenada $\xi_{D}$ determina el orden de la clase a la que dicho punto pertenece (Castañeda \& Muñoz, 2016). El término "componente" se refiere al hecho de que los emisores puntuales virtuales se caracterizan por una expansión modal sobre las clases de pares de emisores, especificadas por su vector de separación. Además, a cada punto se le asigna el valor del coeficiente de la expansión modal de $S_{D}\left(\mathbf{r}_{A}\right)$, como se estipula en la siguiente definición matemática:

$$
\mathbf{S C}_{M}\left(\xi_{A}, \xi_{D}\right)=\left\{S_{M}\left(\xi_{A}\right),\left|\mu_{M}\left(\xi_{+}, \xi_{-}\right)\right| \sqrt{S_{M}\left(\xi_{+}\right)} \sqrt{S_{M}\left(\xi_{-}\right)}\right\}
$$
con

$$
\mathbf{S C}_{M}^{(R)}\left(\xi_{A}, 0\right)=\left\{S_{M}\left(\xi_{A}\right)\right\}
$$

como la clase de orden cero, que se corresponde con el subconjunto de emisores puntuales reales en los pinholes de la rejilla, $\mathrm{y}$

$\mathbf{S C}_{M}^{(V)}\left(\xi_{A}, \xi_{D}^{(n)}\right)=\left\{\left|\mu_{M}\left(\xi_{+}^{(n)}, \xi_{-}^{(n)}\right)\right| \sqrt{S_{M}\left(\xi_{+}^{(n)}\right)} \sqrt{S_{M}\left(\xi_{-}^{(n)}\right)}, n=1,2,3, \cdots\right\}(9 \mathrm{c})$, como la clase de orden $n$, compuesta por el subconjunto de parejas de pinholes con vectores de separación $\xi_{D}^{(n)}$, cuyos puntos medios están ubicados en las posiciones $\xi_{A}$. Las clases están ordenadas de manera que $\left|\xi_{D}^{(n)}\right|<\left|\xi_{D}^{(n+1)}\right|$. Si una pareja de una clase dada está inscrita en un cono de correlación preparado en la etapa SM, la pareja estará correlacionada y será componente del emisor puntual virtual asociado a dicho cono de correlación. Debe subrayarse que cada cono de correlación inscribe, como máximo, un elemento de cada clase correlacionada.

En la interferencia con ondas se ha establecido que la clase de orden cero es necesariamente discreta (Castañeda, 2017 b), y ese atributo es crucial para la caracterización adecuada de fuentes efectivas extendidas y rejillas. En efecto, se reportaron los siguientes criterios para un arreglo lineal regular, con período $b$, de $N$ emisores puntuales reales correlacionados:

$$
\begin{array}{cc}
b>\lambda & \text { interferencia } \\
\lambda / 10<b \leq \lambda<(N-1) b & \text { difracción } \\
\lambda / 10<b<(N-1) b \leq \lambda & \text { transición } \\
(N-1) b \leq \lambda / 10 & \text { emisor puntual real }
\end{array}
$$

El término "transición” en los anteriores criterios indica que ese rango se caracteriza por producir distribuciones de energía de forma acampanada, diferentes tanto a los patrones de difracción convencionales, caracterizados por un máximo central y máximos secundarios laterales, como al patrón lorentziano característico de los emisores puntuales reales. Además, el último criterio, que caracteriza precisamente la producción de dicho patrón lorentziano, es independiente del número $N$ de emisores puntuales reales y de su estado de correlación. Así, en la etapa de preparación SM el segundo criterio caracteriza los conos de correlación y de energía asociados a las fuentes efectivas extensas, en tanto que en la etapa de realización MD el primero caracteriza los conos de energía, en sus componentes real y modulador, asociados a rejillas ubicadas en el plano $\mathrm{M}$.

La Figura 3 muestra el efecto del tamaño de la fuente efectiva uniforme en el plano $\mathrm{S}$, especificado por su longitud $(N-1) \lambda / 2$, sobre la preparación del experimento en la etapa SM. Los perfiles en (a) confirman la reducción en el diámetro del soporte estructurado en el plano $\mathrm{M}$ en la medida en que la longitud de la fuente efectiva aumenta. No obstante, los perfiles en (b) muestran que la sección transversal del cono lorentziano de energía es invariante con respecto al aumento de longitud de la fuente efectiva. Esto se debe a que, (i) los emisores puntuales reales son no-correlacionados, y (ii) la longitud $z^{\prime}$ de la etapa SM es suficientemente larga para que los conos lorentzianos asociados a cada emisor puntual individual se solapen, produciendo un cono resultante con sección transversal lorentziana.

Analicemos ahora las interacciones entre los emisores puntuales reales y virtuales distribuidos en el plano $\mathrm{M}$ de un experimento de interferencia con rejilla, preparado bajo las condiciones ilustradas en la Figura 3. Con el fin de comprender los fundamentos de la construcción del mapa de interacciones con base en el espectro de clases, consideremos inicialmente la realización experimental más simple, que consiste en insertar una rejilla con tres pinholes colineales y equidistantes, de manera que

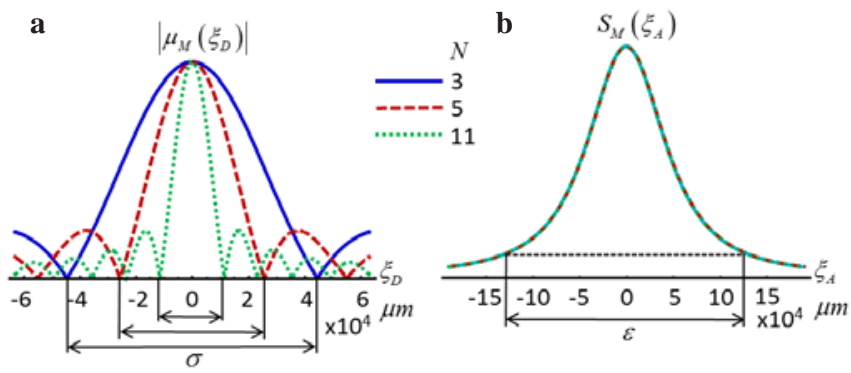

Figura 3. Perfiles de: (a) la magnitud de los grados de correlación en el plano M preparados en la etapa SM considerando como fuente efectiva un arreglo lineal de $N$ emisores puntuales reales idénticos, no-correlacionados y regularmente espaciados con período $\lambda / 2\left(z^{\prime}\right.$ $=10^{5} \lambda, \lambda=632 \mathrm{~nm}$ ). Independientemente de sus tamaños, todas las fuentes efectivas producen la misma distribución lorentziana de energía en el plano $\mathrm{M}$, como muestran los perfiles en (b). Aunque el diámetro del soporte estructurado $\sigma$ disminuye cuando el número de emisores puntuales reales aumenta, el diámetro $\varepsilon$ del área iluminada en el plano M permanece prácticamente invariante. 
$t_{M}\left(\xi_{+}\right) t_{M}\left(\xi_{-}\right)=\left[\delta\left(\xi_{A}+\mathbf{b}\right)+\delta\left(\xi_{A}\right)+\delta\left(\xi_{A}-\mathbf{b}\right)\right] \delta\left(\xi_{D}\right)$

$+\left[\delta\left(\xi_{A}+\mathbf{b} / 2\right)+\delta\left(\xi_{A}-\mathbf{b} / 2\right)\right]\left[\delta\left(\xi_{D}+\mathbf{b}\right)+\delta\left(\xi_{D}-\mathbf{b}\right)\right] \cdot$

$+\left[\delta\left(\xi_{D}+2 \mathbf{b}\right)+\delta\left(\xi_{D}-2 \mathbf{b}\right)\right] \delta\left(\xi_{A}\right)$

En la ecuación (10) es evidente que $t_{M}\left(\xi_{+}\right) t_{M}^{*}\left(\xi_{-}\right)$ introduce las clases de emisores puntuales en el modelo matemático presentado. En general, el producto $t_{M}\left(\xi_{+}\right) t_{M}^{*}\left(\xi_{-}\right)$ determina el máximo número de clases y su distribución en el plano M, el cual podrá ser filtrado por el grado de correlación preparado en la etapa SM. En el caso de una máscara con tres pinholes colineales, la ecuación (10) indica la existencia de un máximo de tres clases de emisores puntuales:

(i) la clase de orden cero (primera línea de la ecuación), conformada por los emisores puntuales reales en los pinholes de la máscara, ubicados en $\xi_{A}=0, \pm \mathbf{b}$;

(ii) la clase de primer orden (segunda línea de la ecuación), caracterizada por el vector de separación $\boldsymbol{\xi}_{D}= \pm$ b. Sus dos miembros determinan dos emisores puntuales virtuales ubicados en los puntos medios entre los pinholes, $\boldsymbol{\xi}_{\mathrm{A}}= \pm \mathbf{b} / 2, \mathrm{y}$

(iii) la clase de segundo orden (tercera línea de la ecuación), con un único miembro con vector de separación $\boldsymbol{\xi}_{D}= \pm 2 \mathbf{b}$, que determina al emisor puntual virtual ubicado en $\boldsymbol{\xi}_{A}=0$, posición compartida con un emisor puntual real.

Por lo tanto, según las ecuaciones (9), el espectro de clases de emisores puntuales para el experimento considerado estará dado por los conjuntos

$$
\mathbf{S C}_{M}^{(R)}(\{ \pm \mathbf{b}, 0\}, 0)=\left\{S_{M}(\mathbf{b}), S_{M}(0), S_{M}(-\mathbf{b})\right\}
$$

para la clase de orden cero,

$$
\begin{array}{r}
\mathbf{S C}_{M}^{(V)}( \pm \mathbf{b} / 2, \mathbf{b})= \\
\left|\mu_{M}(\mathbf{b}, 0)\right| \sqrt{S_{M}(\mathbf{b})} \sqrt{S_{M}(0)}, \\
\left.\left|\mu_{M}(0,-\mathbf{b})\right| \sqrt{S_{M}(0)} \sqrt{S_{M}(-\mathbf{b})}\right\}
\end{array}
$$

para la clase de primer orden, $\mathrm{y}$

$$
\mathbf{S C}_{M}^{(V)}(0,2 \mathbf{b})=\left\{\left|\mu_{M}(\mathbf{b},-\mathbf{b})\right| \sqrt{S_{M}(\mathbf{b})} \sqrt{S_{M}(-\mathbf{b})}\right\}
$$

para la clase de segundo orden. Este espectro de clases se ilustra en la $\mathrm{F}_{\text {ig }}$ ura 4 para una correlación completa en (a1), una correlación parcial en (a2) y no-correlación en (a3). La simetría de los grafos respecto al eje $\xi \mathrm{D}=0$ es redundante y representa solo los dos grados de li $\boldsymbol{b}_{\text {ert }} a_{\mathrm{d}}$ en orientación de los vectores de separación. Debido a la redundancia de esta simetría, basta con considerar el segmento de los espectros de $\xi \mathrm{D} \geq 0$ para construir el mapa completo de interacciones, representado por las flechas en la Figura 4. Estas indican que, en el caso completamente correlacionado, cada emisor puntual real tiene dos interacciones con emisores puntuales virtuales. Específicamente, el emisor puntual real en el pinhole central interactúa con los dos emisores puntuales virtuales de la clase de primer orden, mientras que los emisores puntuales reales en los pinholes de los extremos interactúan con el emisor puntual virtual de la clase de segundo orden y con uno de la clase de primer orden.

Los conos en la fila (1) presentan modulación de alto contraste debido a que todos los miembros de ambas clases (órdenes 1 y 2) contribuyen al potencial geométrico, lo que produce una fuerte interacción; este contraste baja significativamente en los conos de la fila (2), puesto que la filtración de clases debilita los emisores puntuales virtuales y sus contribuciones al potencial geométrico, lo cual a su vez debilita las interacciones; por último, la fila (3) no incluye conos modulados porque todas las clases han sido filtradas, de manera que el potencial geométrico desaparece $\mathrm{y}$ no se producen interacciones. Debe anotarse que el cono $\mathbf{W}_{M D}^{(-)}\left(\xi_{A} ; \mathbf{r}_{A}\right)$ no se incluye porque es el simétrico de $\mathbf{W}_{M D}^{(+)}\left(\boldsymbol{\xi}_{A} ; \mathbf{r}_{A}\right)$ (columna (b)) con respecto al eje $\boldsymbol{\xi}_{A}=\mathrm{r}_{A}=0$.

El cono modulado $\mathbf{W}_{M D}\left(\xi_{A} ; \mathbf{r}_{A}\right)=\mathbf{W}_{M D}^{(+)}\left(\xi_{A} ; \mathbf{r}_{A}\right)+\mathbf{W}_{M D}^{(0)}\left(\xi_{A} ; \mathbf{r}_{A}\right)+$ $\mathbf{W}_{M D}^{(-)}\left(\xi_{A} ; \mathbf{r}_{A}\right)$, representado en los grafos de la columna (d), indica el resultado de un número significativo de realizaciones de las seis interacciones que se producen en este interferómetro. La distribución de energía del patrón de interferencia registrado por el detector corresponde a la sección transversal de este cono en el plano $\mathrm{D}$, dada por $S_{D}\left(\mathbf{r}_{A}\right)=S_{D}^{(+)}\left(\mathbf{r}_{A}\right)+S_{D}^{(0)}\left(\mathbf{r}_{A}\right)+S_{D}^{(-)}\left(\mathbf{r}_{A}\right)$, como lo ejemplifica el perfil vertical para $z=10 \lambda$ en dichos grafos.

Como consecuencia de las interacciones, la energía de las ondas que se mueven en la etapa MD es dirigida hacia las franjas brillantes de los grafos de la Figura 4, las cuales satisfacen las condiciones $S_{D}^{( \pm)}\left(\mathbf{r}_{A}\right)>0, S_{D}^{(0)}\left(\mathbf{r}_{A}\right)>0$ $\mathrm{y}$, por lo tanto, $S_{D}\left(\mathbf{r}_{A}\right)>0$. Los máximos de estas franjas brillantes caracterizan lo que denominamos "interferencia constructiva”. Las regiones en las que estas condiciones no se cumplen son regiones prohibidas para el movimiento de las ondas. En particular, las franjas para las que $S_{D}^{( \pm)}\left(\mathbf{r}_{A}\right)=0$, $S_{D}^{(0)}\left(\mathbf{r}_{A}\right)=0 \mathrm{y}$, por lo tanto, $S_{D}\left(\mathbf{r}_{A}\right)=0$, caracterizan lo que denominamos "interferencia destructiva".

Las imágenes de la fila (1) de la Figura 4 ilustran la interferencia bajo correlación completa, mientras que las de la fila (2) muestran el efecto de la correlación parcial debida a la filtración de clases realizada por un grado de correlación de la forma $\mu_{M}\left(\xi_{D}\right)=\exp \left(-\left|\xi_{D}\right|^{2} / 2 \sigma^{2}\right)$, con $\sigma=1.5 \lambda$. En este caso, la clase de segundo orden es removida del espectro de clases de emisores puntuales y el potencial geométrico aportado por la clase de primer orden se debilita. En consecuencia, se producen apenas cuatro tipos de interacciones de menor energía que las de la correlación completa, de manera que la modulación de franjas de los conos pierde contraste y las franjas de los patrones de interferencia pierden visibilidad. Para la fila (3) se tiene que $\sigma=0.3 \lambda$, con lo cual el grado de correlación filtra en su totalidad las clases de primero y segundo órdenes. Así, tanto el potencial geométrico como las interacciones desaparecen y solo se mantienen los conos lorentzianos asociados a los emisores puntuales reales en los pinholes de la máscara en el plano M. Estos conos determinan la geometría para el movimiento de las ondas en la etapa MD, por lo cual el patrón registrado en el plano D no presenta franjas de interferencia.

El procedimiento anterior constituye el elemento algorítmico básico para explicar y predecir la interferencia tanto de ondas como de partículas masivas, con un número arbitrario de emisores puntuales reales en el plano $\mathrm{M}$ y en arreglos 

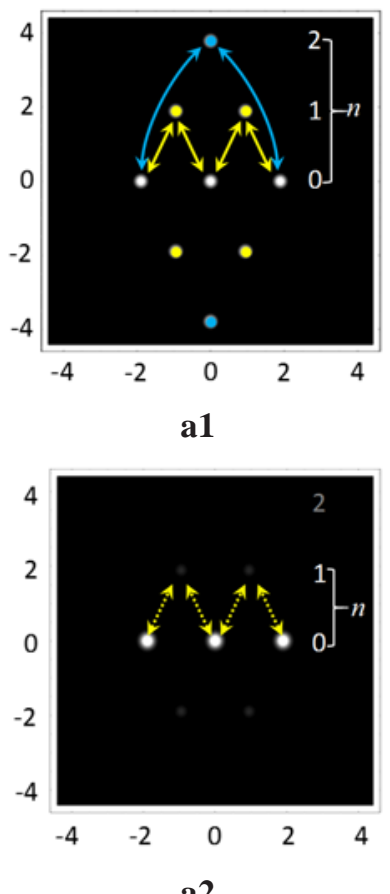

a2

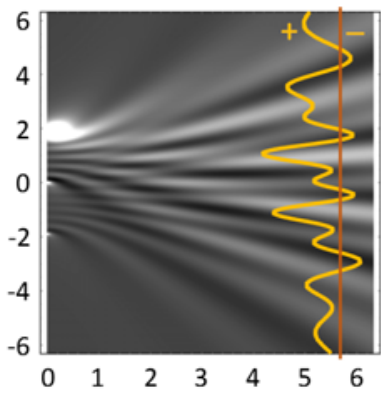

b1

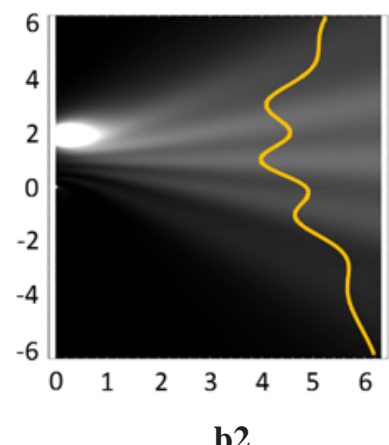

b2

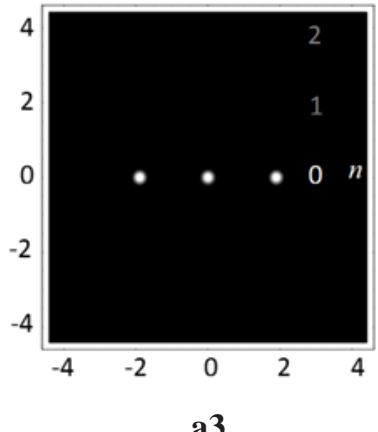

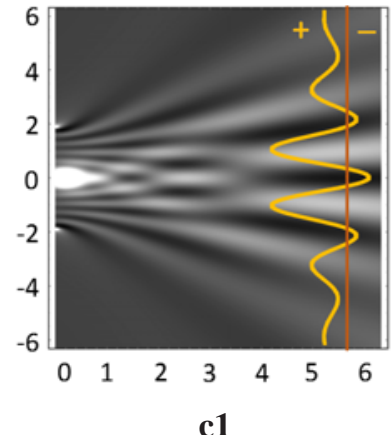

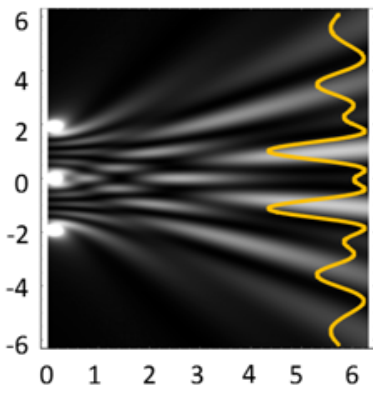

d1
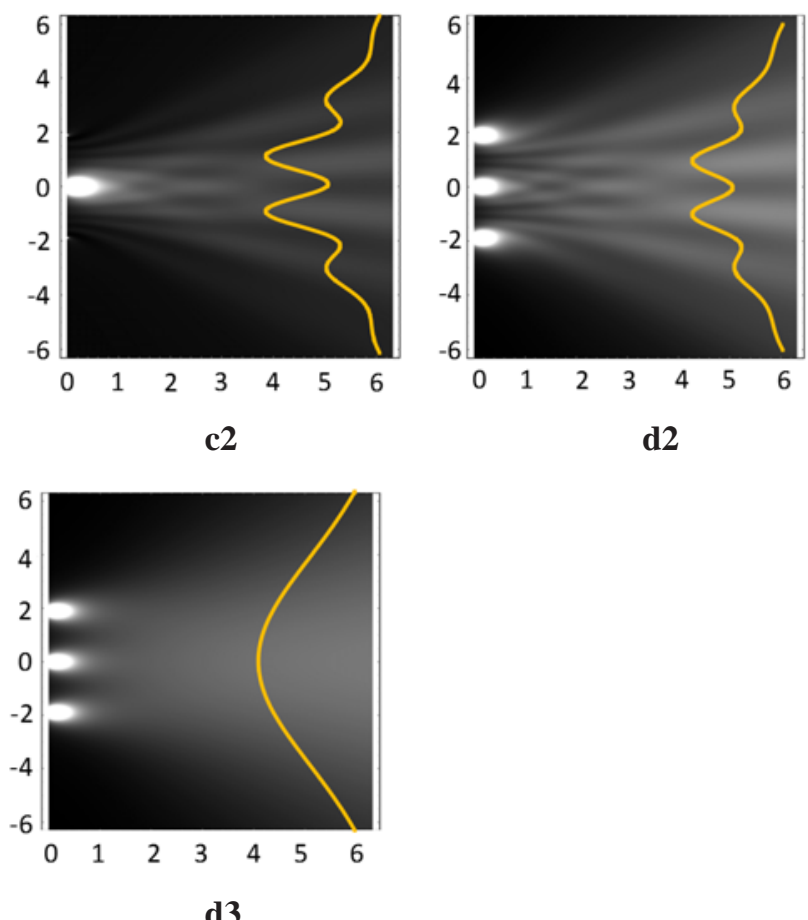

Figura 4. Potencial geométrico para la interferencia de ondas $(\lambda=632 \mathrm{~nm})$ en un interferómetro con máscara de tres pinholes colineales, con espaciamiento uniforme de $3 \lambda$, bajo correlación completa en la fila (1), correlación parcial (grado de correlación gaussiano con desviación estándar de 1.5 $\lambda$ ) en la fila (2) y no-correlación (grado de correlación gaussiano con desviación estándar de $0.3 \lambda$ ) en la fila (3). La columna (a) muestra los grafos de los espectros de clases de emisores puntuales, donde el índice $n$ denota el orden de las clases y las flechas indican las interacciones entre cada emisor puntual real y los emisores puntuales virtuales. Sus ejes horizontal y vertical son $\boldsymbol{\xi}_{A} \mathrm{y}$ $\boldsymbol{\xi}_{D}$, y sus unidades son $\mu m$.

experimentales caracterizados por conjuntos arbitrarios de emisores puntuales virtuales. Esto se ilustra en la Figura 5 para la interferen cia con ondas y partículas masivas, considerando un máximo de 11 clases $(0 \leq \mathrm{n} \leq 10)$, cada una con 11-n elementos. Las flechas signadas como interaction links directions (ILD) son clave para la construcción del mapa de interacciones en cada caso. En efecto, cada emisor puntual real interactúa solo con los elementos de las clases que son colineales con él a lo largo de las direcciones marcadas por las flechas ILD. Así, el mapa de interacciones se construye colocando la punta inferior de ambas flechas en cada elemento de la clase de orden cero para vincularlo con los puntos del espectro a lo largo de las flechas, como lo indican las rectas punteadas en (a1) y (a2). El vínculo de un elemento de la clase de orden cero con un elemento dado de una clase de orden mayor que cero representa una interacción, descrita por la nueva ley general de interferencia. El mapa de interacciones completo queda construido una vez que este procedimiento se aplique a todos los elementos de la clase de orden cero.

El ejemplo de la fila (1) en la Figura 5 indica que cada emisor puntual real interactúa con componentes de 10 emisores puntuales virtuales, aunque el número de emisores puntuales en este caso es 19. Este número de interacciones se reduce en los ejemplos de las filas (2) y (3), y las interacciones se debilitan, a su vez, debido al debilitamiento del potencial geométrico causado por la filtración de clases, lo cual se consigue aumentando el tamaño de la fuente efectiva 


$$
\lambda=632 \mathrm{~nm}
$$
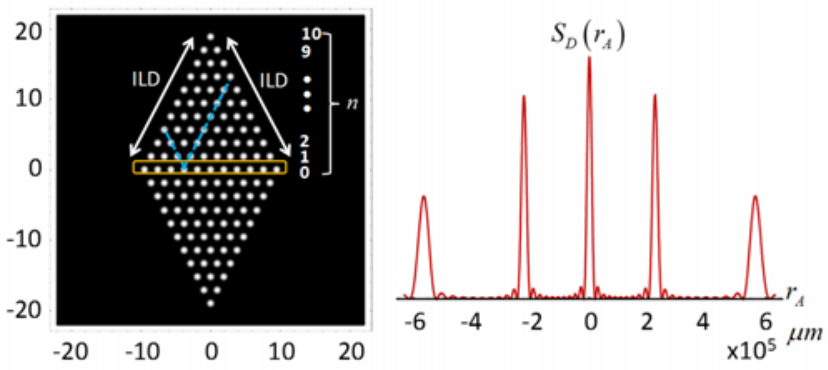

a1

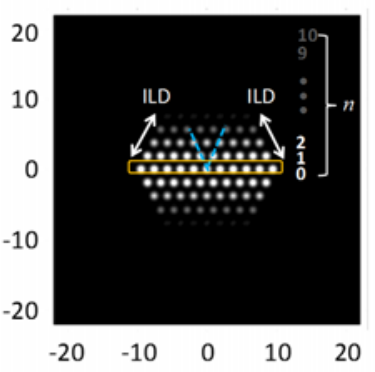

$\mathbf{a} 2$

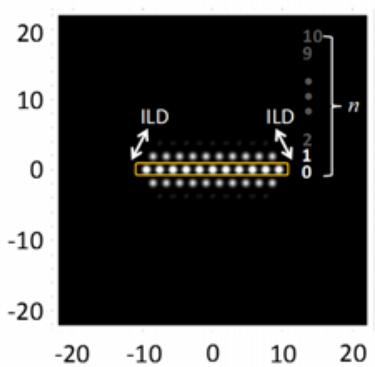

a3

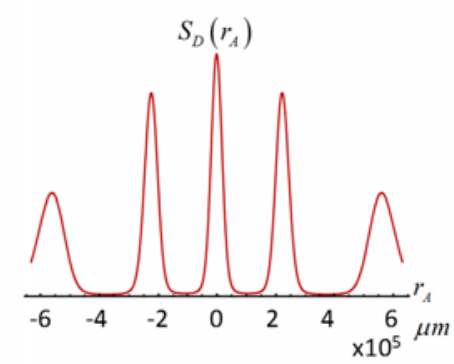

b2

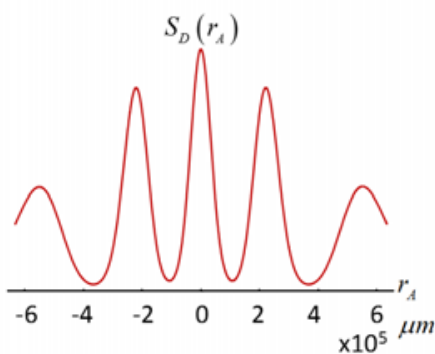

b3

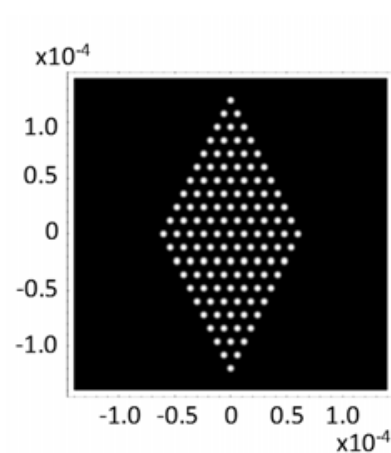

c1

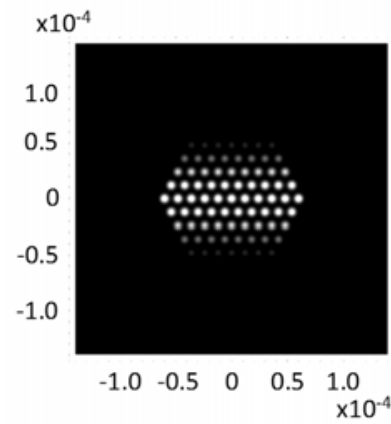

c2

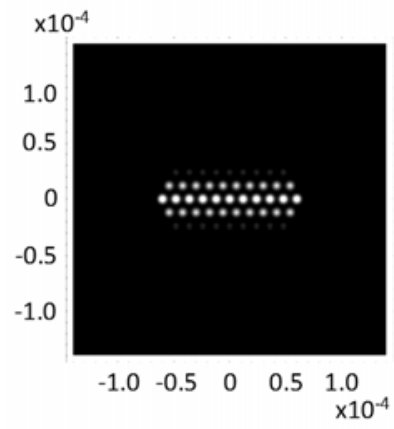

c3 $\lambda=4 \mathrm{pm}$

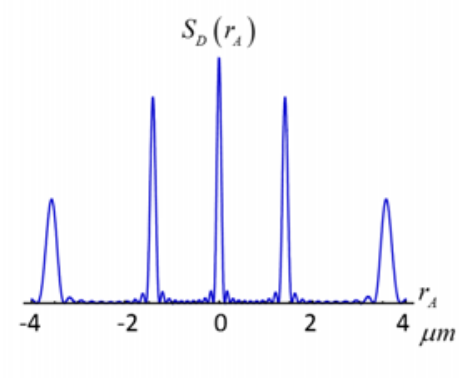

d1

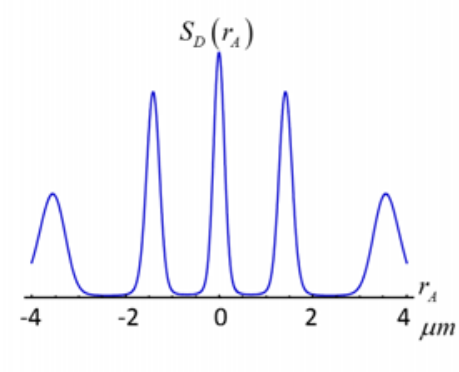

d2

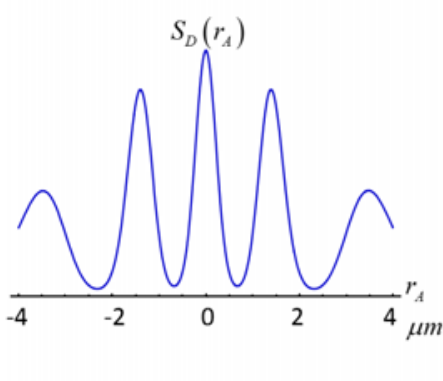

d3

Figura 5. Espectros de clases de emisores puntuales y sus patrones de interferencia de campo lejano $\left(z=10^{6} \lambda\right)$ para interferencia de ondas y partículas masivas con rejilla de 11 pinholes, espaciamiento de $3 \lambda$, y bajo grado de correlación gaussiana. Los espectros de clases en la columna (a) muestran la clase de orden cero encerrada en recuadro. Sus emisores puntuales reales interactúan solo con las componentes de los emisores puntuales virtuales a lo largo de las flechas ILD, como se muestra con las líneas punteadas. El tamaño de los soportes estructurados es mayor que la longitud $30 \lambda$ de la rejilla en la fila (1), indicando que el orden máximo de las clases es 10 en los espectros (a1) y (a3). Las interacciones producen los patrones (a2) y (a4), respectivamente, cuyos máximos principales son estrechos y de alto contraste, separados por máximos secundarios de baja energía. El tamaño de los soportes estructurados es menor que la longitud de la rejilla en las filas (2) y (3), por lo que las familias de orden superior son filtradas, lo que ensancha los máximos principales, elimina los secundarios y reduce el contraste de los patrones. Las posiciones de los máximos principales son invariantes. El eje horizontal $\boldsymbol{\xi}_{A}$ y el vertical $\boldsymbol{\xi}_{D}$ de los espectros de clases de emisores puntuales están en $\mu m$.

en el plano S. Específicamente, las clases para $n \geq 4$ en la fila (2) y para $n \geq 3$ en la fila (3) se suprimen. Esto ensancha los máximos principales, elimina los secundarios y reduce el contraste de los patrones correspondientes.

\section{Resumen y conclusiones}

El presente análisis da respuesta a la pregunta expresada en su título: la superposición de ondas no se requiere ni puede constituir el principio generalizado desde el que se explique, de manera unificada, la interferencia de ondas clásicas y de partículas masivas. En el marco de la teoría clásica de la coherencia óptica, se sintetizó la interacción entre emisores puntuales reales y virtuales como principio unificado de interferencia, así como la nueva ley general de interferencia. Este principio es diferente e incompatible con la superposición de ondas y se apoya en una descripción geométrica novedosa de la configuración y la operación de cualquier experimento de interferencia con ondas o con 
partículas singulares. Los aspectos más destacables de esta descripción son: (i) la interpretación geométrica de la correlación en la etapa de preparación del experimento, que explica, además, la fenomenología involucrada en el teorema de Van Cittert - Zernike, y (ii) la caracterización del experimento en términos de conjuntos de fuentes puntuales reales y virtuales, representando el primero las ondas o las partículas que se mueven en el interferómetro, mientras que el segundo caracteriza al interferómetro mismo, generando en la etapa de realización del experimento el potencial geométrico responsable de la redistribución de la energía de las ondas y las partículas en patrones con franjas.

Se demostró, asimismo, cómo el principio unificado de interferencia se aplica a cualquier experimento de interferencia mediante la herramienta "espectro de clases de emisores puntuales", la cual proporciona el mapa completo de las interacciones que ocurren en un experimento específico. El modelo completo se ha validado mediante el análisis y predicción de patrones de interferencia en experimentos con ondas de luz (Castañeda, 2017 a), electrones singulares y moléculas de $\mathrm{C}_{60}, \mathrm{PcH}_{2}$ y $\mathrm{F}_{24} \mathrm{PcH}_{2}$ reportados por Nairz, et al., 2003 y Juffmann, et al., 2012, respectivamente, discutidos en detalle en Castañeda, et al., 2016 a, b.

Aunque el impacto inmediato de las ideas expresadas en este trabajo atañe al diseño de experimentos de interferencia, particularmente en la escala de la microtecnología y la nanotecnología (Castañeda, et al., 2016 a, b; Castañeda, 2014; Castañeda, 2017 a), su impacto más significativo y profundo está en el planteamiento de una concepción alternativa de los fundamentos mismos de un fenómeno básico para la física como es la interferencia. Esta concepción configura un escenario de convergencia de las descripciones clásica y cuántica del fenómeno, restableciendo lo estipulado en las reglas de la filosofía natural de Newton que aún rigen para la reflexión en física. Sin embargo, debido a lo novedoso de su terminología y al uso de conceptos no estandarizados, abrirá también nuevos campos de discusión sobre los fundamentos físicos de este fenómeno.

\section{Referencias}

Arndt, M., Nairz, O., Vos-Andreae, J., Keller, C., van der Zouw, G., and Zeilinger, A. (1999). Wave-particle duality of $\mathrm{C}_{60}$ Molecules. Nature. 401: 680-682.

Bach, R., Pope, D., Liou, S.H., Batelaan, H. (2013). Controlled double-slit electron diffraction. New J. Phys. 15: 033018 (7pp).

Born, M., and Wolf, E. (1993). Principles of Optics (6th ed. Pergamon Press: Oxford). p. 836.
Castañeda, R. (2014). Electromagnetic wave fields in the microdiffraction domain. Phys. Rev. 89: 013843 (14pp).

Castañeda, R., Matteucci, G., Capelli, R. (2016). Quantum Interference without Wave-Particle Duality. J. Mod. Phys. 7: 375-389.

Castañeda, R., Matteucci, G., Capelli, R. (2016). Interference of Light and of Material Particles: A Departure from the Superposition Principle. Hawkes, P.H. editor, Advances in Imaging and Electron Physics, Vol. 197, Burlington: Academic Press, p. 1-43.

Castañeda, R., Muñoz, H. (2016). Spectrum of classes of point emitters: New tool for nonparaxial optical field modeling. J. Opt. Soc. Am. 33: 1421-1429.

Castañeda, R. (2017). Interaction description of light propagation. J. Opt. Soc. Am. 34: 1035-1044.

Castañeda, R. (2017). Discreteness of the real point emitters as a physical condition for diffraction. J. Opt. Soc. Am. 34: 184-192.

Feynman, R. P., Leighton R.B., and Sands, M. (1965). The Feynman Lectures on Physics vol 3. Addison-Wesley: Menlo Park, CA.

Feynman, R. P., Hibbs, A. R. (1965). Quantum Mechanics and Path Integrals. McGraw-Hill: New York. p. 365.

Frabboni, S., Gabrielli, A., Gazzadi, G.C., Giorgi, F., Matteucci, G., Pozzi, G., Cesari, N.S., Villa, M., Zoccoli,A.(2012). The Young-Feynman two-slit experiment with single electrons: Build-up of the interference pattern and arrival-time distribution using a fast-readout pixel detector. Ultramicroscopy. 116: $73-76$

Gaskill, J. (1978). Linear systems, Fourier transforms and Optics. John Wiley \& Sons: New York. p. 554.

Juffmann, T., Truppe, S., Geyer, P., Major, A.G., Deachapunya, S., Ulbricht, H., Arndt, M. (2009). Wave and particle in molecular interference lithography. Phys. Rev. Lett. 103: 263601-1 - 263601-4.

Juffmann, T., Milic, A., Muellneritsch, M., Asenbaum, P., Tsukernik, A., Tuexen, J., Mayor, M., Cheshnovsky, O., Arndt, M. (2012). Real-time single-molecule imaging of quantum interference. Nature Nanotech. 7: 297-300.

Mandel, L., Wolf, E. (1995). Optical Coherence and Quantum Optics. Cambridge University Press: Cambridge. p. 1166.

Matteucci, G., Pezzi, M., Pozzi, G., Alberghi, G., Giorgi, F., Gabrielli, A., Semprini, N., Villa, M., Zoccoli, A., Frabboni, S., Gazzadi, G. (2013). Build-up of interference patterns with single electrons. Eur. J. of Phys. 34: 511-517.

Nairz, O., Arndt, M., Zeilinger, A. (2003). Quantum interference experiments with large molecules. Am. J. Phys. 71: 319-325.

Newton, I. (1687). Principios matemáticos de la Filosofía natural [Philosophiae Naturalis Principia Mathematica]. Ediciones Altaya, S.A. Grandes Obras del Pensamiento, 21 (1993): Barcelona, p. 621.

Zeilinger, A., Gaehler, R., Shull, C.G., Treimer, W., Mampe, W. (1988). Single and double-slit diffraction of neutrons. Rev. Mod. Phys. 60:1067-73. 